\title{
Emergence of Cracklike Behavior of Frictional Rupture: The Origin of Stress Drops
}

\author{
Fabian Barras $\odot,{ }^{1}$ Michael Aldam $\odot,{ }^{2}$ Thibault Roch $\odot,{ }^{1}$ Efim A. Brener $\odot,{ }^{3,4}$ \\ Eran Bouchbinder $\odot^{2, *}$ and Jean-François Molinari ${ }^{1, \dagger}$ \\ ${ }^{1}$ Civil Engineering Institute, Materials Science and Engineering Institute, \\ Ecole Polytechnique Fédérale de Lausanne, Station 18, CH-1015 Lausanne, Switzerland \\ ${ }^{2}$ Chemical and Biological Physics Department, Weizmann Institute of Science, Rehovot 7610001, Israel \\ ${ }^{3}$ Peter Grünberg Institut, Forschungszentrum Jülich, D-52425 Jülich, Germany \\ ${ }^{4}$ Institute for Energy and Climate Research, Forschungszentrum Jülich, D-52425 Jülich, Germany
}

(Received 18 June 2019; revised manuscript received 2 September 2019; published 26 November 2019)

The process of frictional rupture, i.e., the failure of frictional systems, abounds in the technological and natural world around us, ranging from squealing car brake pads to earthquakes along geological faults. A general framework for understanding and interpreting frictional rupture commonly involves an analogy to ordinary crack propagation, with far-reaching implications for various disciplines from engineering tribology to geophysics. An important feature of the analogy to cracks is the existence of a reduction in the stress-bearing capacity of the ruptured interface, i.e., of a drop from the applied stress, realized far ahead of a propagating rupture, to the residual stress left behind it. Yet, how and under what conditions such finite and well-defined stress drops emerge from basic physics are not well understood. Here, we show that for a rapid rupture a stress drop is directly related to wave radiation from the frictional interface to the bodies surrounding it and to long-range bulk elastodynamics and not exclusively to the physics of the contact interface. Furthermore, we show that the emergence of a stress drop is a transient effect, affected by the wave travel time in finite systems and by the decay of long-range elastic interactions. Finally, we supplement our results for rapid rupture with predictions for a slow rupture. All of the theoretical predictions are supported by available experimental data and by extensive computations. Our findings elucidate the origin of stress drops in frictional rupture; i.e., they offer a comprehensive and fundamental understanding of why, how, and to what extent frictional rupture might be viewed as an ordinary fracture process.

DOI: 10.1103/PhysRevX.9.041043

Subject Areas: Geophysics, Mechanics, Soft Matter

\section{BACKGROUND AND MOTIVATION}

Rapid slip along interfaces separating bodies in frictional contact is mediated by the spatiotemporal dynamics of frictional rupture [1,2]. Frictional rupture is a fundamental process of prime importance for a broad range of physical systems; e.g., it is responsible for squealing in car brake pads [3], for bowing on a violin string [4], and for earthquakes along geological faults [5-7], to name just a few well-known examples. The essence of frictional rupture propagation is that a state of relatively high slip rate (the rate of interfacial shear displacement discontinuity) behind the rupture edge propagates into a low or vanishing slip-rate state ahead of it; cf. Fig. 1. As such, frictional rupture

\footnotetext{
*eran.bouchbinder@weizmann.ac.il

†jean-francois.molinari@epfl.ch
}

Published by the American Physical Society under the terms of the Creative Commons Attribution 4.0 International license. Further distribution of this work must maintain attribution to the author(s) and the published article's title, journal citation, and DOI. appears to be essentially similar to ordinary tensile (opening) cracks, where a finite tensile displacement discontinuity (broken material) state behind the crack edge propagates into a zero tensile displacement discontinuity (intact material) state ahead of it [8].

There is, however, an important and fundamental difference between frictional rupture and ordinary tensile cracks that manifests itself in the stress states associated with these two processes. A tensile crack, i.e., a crack subjected to opening forces, is composed of surfaces that cannot support stress, so the stress behind its edge vanishes. Consequently, tensile (opening) crack propagation is a process in which the applied stress ahead of the crack edge drops to zero behind it. This stress drop, which accompanies tensile crack propagation, has dramatic implications. Most notably, the loss of stress-bearing capacity along the crack surfaces is compensated by a large concentration of deformation and stress near the crack edge, oftentimes in a way that mimics a mathematical singularity, whose intensity increases with increasing stress drops [8]. Frictional rupture is different from tensile cracks, because the finite frictional interaction between the two bodies in contact behind the rupture edge 


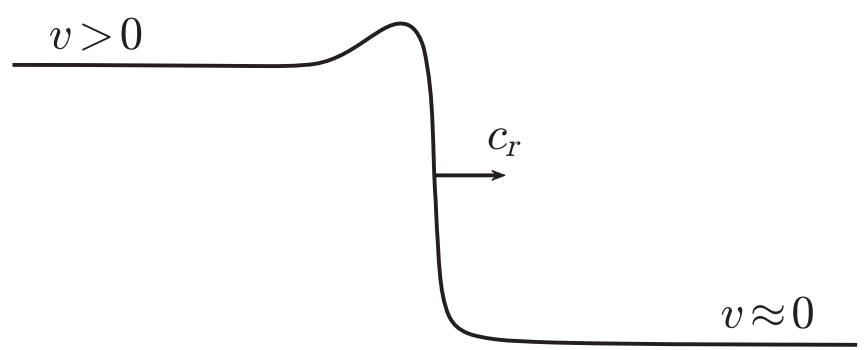

FIG. 1. A schematic representation of the spatial slip-rate $v$ profile of a frictional rupture propagating at a velocity $c_{r}$ from left to right. A slipping or sliding state with a relatively high slip velocity $v>0$ characterizes the interface behind the propagating rupture edge, and a low or vanishing slip-rate state, $v \approx 0$, characterizes the interface ahead of it.

generically implies that the stress there cannot drop to zero but rather remains finite.

The close relations between frictional rupture and tensile cracks can be maintained if, as is widely assumed, the stress behind the frictional rupture edge- the residual stress $\tau_{\text {res }}$-is well defined and is generically smaller than the far-field stress $\tau_{d}$ that is required to drive a rupture. Moreover, the residual stress $\tau_{\text {res }}$ is generally assumed to be an intrinsic interfacial property of the slipping contact interface, typically related to the kinetic friction coefficient. Under these assumptions, a finite stress drop $\Delta \tau \equiv \tau_{d}-$ $\tau_{\text {res }}>0$ exists, and effective cracklike properties of frictional rupture, e.g., edge singularity, are expected to emerge. These assumptions have been adopted in an extremely broad range of theoretical and numerical studies [9-27], and their implications have been consistent with geophysical observations [26,28] and have been confirmed in some recent laboratory experiments [29-36]. In fact, stress drops are among the few remotely observable parameters in earthquake science, providing a key link to the frictional properties of faults, which can be only indirectly inferred.

Yet, to the best of our knowledge, currently there is no basic understanding of how and under what conditions the effective cracklike behavior of frictional rupture emerges from fundamental physics. More specifically, there is a need to understand what the physical origin of a finite stress drop $\Delta \tau>0$ is and under what conditions it emerges. Here, we address these basic questions; first, we show that for a rapid rupture a finite and well-defined stress drop is not an interfacial property, as is widely assumed, but rather it is directly related to wave radiation from the frictional interface to the bodies surrounding it (the so-called radiation damping effect [37-40]) and to long-range bulk elastodynamic interaction effects. Second, we show that the emergence of a stress drop is a finite-time effect, limited by the wave travel time in finite systems. Third, we show that for a slow rupture, i.e., a rupture that is significantly slower than the elastic wave speeds [41-44], stress drops are transiently controlled by the long-range quasistatic elasticity of the bodies surrounding the frictional interface. A reanalysis of very recent experimental results, reported by two different experimental groups, provides strong support to our theoretical predictions, for both a rapid and a slow rupture. All in all, our findings elucidate the origin of stress drops in frictional rupture; i.e., they offer a comprehensive and fundamental understanding of why, how, and to what extent frictional rupture might be viewed as an ordinary fracture process.

\section{THE PHYSICAL ORIGIN AND MAGNITUDE OF THE STRESS DROP ASSOCIATED WITH FRICTIONAL RUPTURE}

The starting point for our discussion is a physically motivated interfacial constitutive law, i.e., a relation between the dynamical and structural variables that characterize a frictional interface and the frictional resistance stress $\tau$ [45]. A frictional interface is formed when two bodies come into contact. Each of them satisfies its own continuum momentum balance equation $\rho \ddot{\boldsymbol{u}}(\boldsymbol{r}, t)=$ $\nabla \cdot \boldsymbol{\sigma}(\boldsymbol{r}, t)$, where $\rho$ is the mass density, $\boldsymbol{u}$ and $\boldsymbol{r}=(x, y)$ (in two dimensions) are the displacement and position vector fields, respectively, and $\sigma$ is the stress tensor field (a superposed dot represents a time derivative). $\sigma$ in each body is related to $\boldsymbol{u}$ through a bulk constitutive law (i.e., a constitutive law that characterizes the bodies forming the interface), oftentimes Hooke's law of linear elasticity, adopted below as well. Note that body forces are neglected in the momentum balance equation.

The interfacial constitutive law involves three bulk quantities evaluated at the interface located at $y=0$ : (i) the slip rate or velocity $v(x, t) \equiv \dot{u}_{x}\left(x, y=0^{+}, t\right)-$ $\dot{u}_{x}\left(x, y=0^{-}, t\right)$, where \pm correspond to the upper and lower bodies, respectively, (ii) the shear stress $\sigma_{x y}(x, y=0, t)$, that is balanced by the frictional stress, $\tau(x, t)=\sigma_{x y}(x, y=0, t)$ and (iii) the normal stress $\sigma(x, t) \equiv-\sigma_{y y}(x, y=0, t)$. A large body of evidence accumulated in the past few decades indicates that the interfacial constitutive law must also involve a set of nonequilibrium order parameters $\left\{\phi_{i}\right\}$, sometimes termed internal-state fields, that represent the structural state of the interface and encode its history [45-52]. In a minimal formulation, adopted in numerous studies [38,39,53-58], a single internal-state field $\phi(x, t)$ is used. This assumption is adopted here, without loss of generality.

The interfacial constitutive law, at any position $x$ along the interface and at any time $t$, is described by the following local relation:

$$
\tau=\sigma \operatorname{sgn}(v) f(|v|, \phi),
$$

which must be supplemented with a dynamical equation for the evolution of $\phi$. Extensive evidence indicates that $\phi$ physically represents the age or maturity of the contact $[45,47-52]$ and that its evolution takes the form 


$$
\dot{\phi}=g\left(\frac{|v| \phi}{D}\right)
$$

with $g(1)=0$ and where $\phi$ is of time dimension. The characteristic slip displacement $D$ controls the transition from a stick state $v \approx 0$, with a characteristic structural state $\phi=\phi_{0}$, to a steadily slipping or sliding state $v>0$, with $\phi_{\mathrm{ss}}=D / v$. The precise functional form of $g(\cdot)$ [with $g(1)=0$ ] plays no role in what follows.

The function $f\left(|v|, \phi_{\mathrm{ss}}=D / v\right)=\tau_{\mathrm{ss}}(v) / \sigma$, under steady-state sliding conditions and a controlled normal stress $\sigma$, has been measured over a broad range of slip rates $v$ for many materials [45]. Together with general theoretical considerations [59], it is now established that the steadystate frictional stress $\tau_{\mathrm{ss}}(v)$ is generically $N$ shaped, as shown in Fig. 2(a). Consider then a frictional system driven by a shear stress $\tau_{d}$, which is larger than the minimum of the $\tau_{\mathrm{ss}}(v)$ curve; cf. Fig. 2(a). What are the generic properties of frictional rupture that might emerge under these conditions?

As explained in Sec. I, frictional rupture is a propagating spatiotemporal object that features a relatively high slip state $v>0$ behind its edge and a stick (no or slow slip) $v \approx 0$ state ahead of it, as shown in Fig. 1. This spatiotemporal dynamical process can be directly related to Fig. 2(a), where the driving stress $\tau_{d}$ intersects the $\tau_{\mathrm{ss}}(v)$ curve at three points. The leftmost intersection point features an extremely small slip velocity $v \approx 0$, which corresponds to the state ahead of the rupture edge. We stress that, in general, the state of the interface ahead of the rupture edge may be far from steady state, and the results to follow are largely independent of its detailed properties. The rightmost intersection point features a relatively large slip velocity $v>0$, which corresponds to the state behind the rupture edge. The transition between these two states takes place in the edge region and is controlled by $\dot{\phi}$ in Eq. (2) and by spatiotemporal bulk dynamics. In this transition region, the slip velocity $v$ also goes through the intermediate intersection point, which is not a stable fixed point as the other two. The crucial observation is that the stress behind a steadily propagating frictional rupture is $\tau_{d}$; i.e., the residual stress equals the driving stress, $\tau_{\text {res }}=\tau_{d}$. This observation implies that we expect no stress drop to emerge at all, $\Delta \tau=\tau_{d}-\tau_{\text {res }}=0$, and consequently no cracklike behavior.

In many studies available in the literature, a steady-state friction curve $\tau_{\mathrm{ss}}(v)$ that does not feature a minimum is adopted [38,39,62-64]. We consequently discuss here such a no-minimum friction curve and plot an example of it in Fig. 2(a). In this case, the driving stress $\tau_{d}$ behind the rupture edge cannot be balanced by the friction stress, and the slip velocity in this region is expected to continuously accelerate. As such, we cannot expect a well-defined steady-state stress drop to emerge, though the stress will definitely drop below $\tau_{d}$ behind the rupture edge.
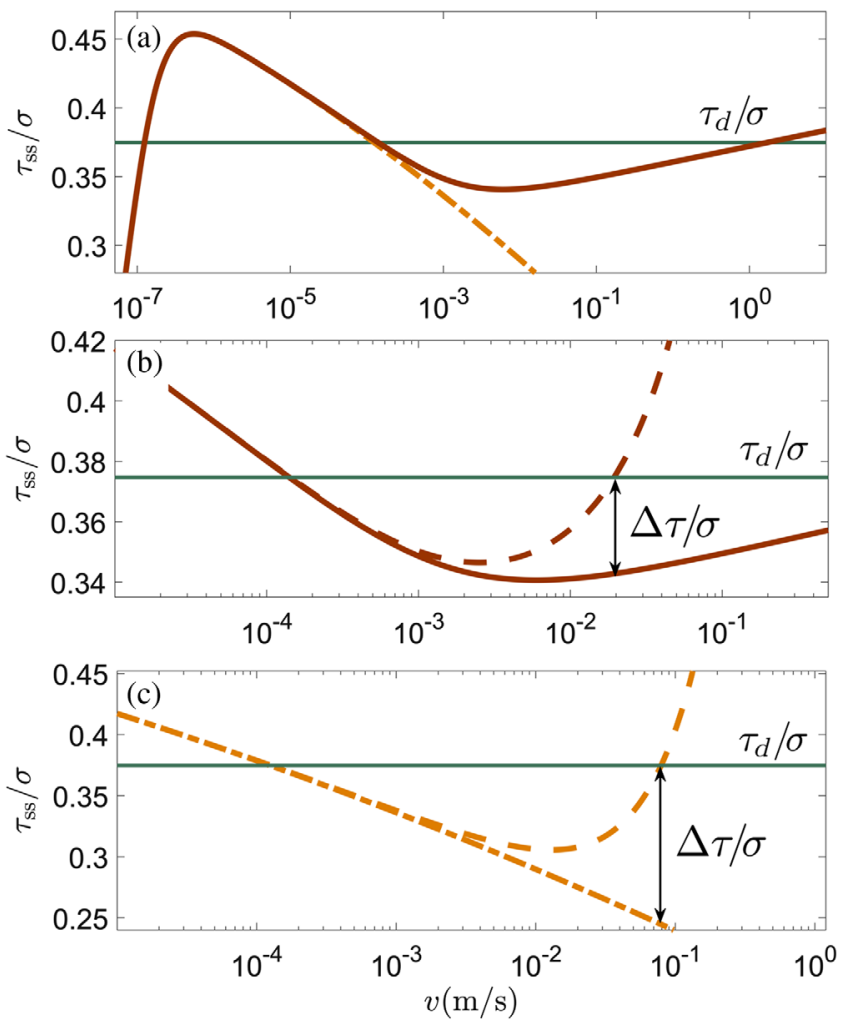

FIG. 2. (a) A schematic representation of the steady-state friction stress $\tau_{\mathrm{ss}}(v)$, normalized by a constant normal stress $\sigma$, versus the slip rate $v$ (solid brown line). The curve has a generic $N$ shape [59], with a maximum at an extremely low $v$ and a minimum at an intermediate $v$. The horizontal line represents the driving stress $\tau_{d}$, which intersects the $N$-shaped steady-state friction curve at three points; the leftmost and rightmost ones are stable fixed points, while the intermediate one is an unstable one. Also shown is a steady-state friction curve without the minimum (dash-dotted orange line) [46,60,61]. This no-minimum steadystate friction curve lacks the rightmost intersection point of the solid brown curve. (b) The effective steady-state friction curve (dashed brown line), obtained by adding $\left(\mu / 2 c_{s}\right) v$ (with $\mu=$ $9 \mathrm{GPa}$ and $c_{s}=2739 \mathrm{~m} / \mathrm{s}$ ) to the solid brown line of (a), together with a copy of the solid brown line of (a) itself. The intersection of the dashed brown line with the horizontal $\tau_{d}$ line [the same as in (a)] is described by Eq. (4), and the stress drop $\Delta \tau$ of Eq. (5) is marked by the black double arrow. (c) The same as (b), but for the no-minimum curve (dash-dotted orange line) of (a).

The discussion above, for both the $N$-shaped and the nominimum steady-state friction curves, seems to lead to the quite remarkable conclusion that based on basic physics considerations we expect no finite and well-defined steadystate stress drops to emerge at all in the context of friction rupture and, hence, no cracklike behavior as well. This conclusion appears to be in sharp contrast to ample evidence indicating the existence of finite and well-defined steady-state stress drops in various frictional systems $[1,29,30,32,33,35,65,66]$. How can one reconcile the two apparently conflicting conclusions? 
To address this question, let us write down in more detail the general expression for interfacial shear stress $\sigma_{x y}(x, y=0, t)$, valid also out of the steady state, when a constant driving stress $\tau_{d}$ is applied at the far boundaries of the systems, say, at $y= \pm H$ ( $H$ is the height of each of the two bodies in contact). For bulk linear elastodynamics, we have $\sigma_{x y}(x, y=0, t)=\tau_{d}+\tilde{s}(x, t)$, where $\tilde{s}(x, t)$ is a spatiotemporal integral that quantifies the long-range (in both space and time) elastodynamic interaction between different parts of the interface [67-69]. Under strict homogeneous steady-state conditions, we have $\tilde{s}(x, t) \rightarrow 0$ and, consequently, $\sigma_{x y}(x, y=0, t) \rightarrow \tau_{d}$, which corresponds to the rightmost intersection point in Fig. 2(a) attained far behind the rupture edge (see the discussion above). At finite times, before strict steady-state conditions are attained, the spatiotemporal integral term $\tilde{s}(x, t)$ makes a finite contribution to $\sigma_{x y}(x, y=0, t)$, which quantifies the deviation from the steady state.

Under these conditions, and, in particular, for times in which information regarding the evolution of the slip velocity $v(x, t)$ relative to some initial or reference slip velocity $v_{0}$ does not have enough time to propagate to the boundaries at $y= \pm H$ and back to the interface, the spatiotemporal integral term $\tilde{s}(x, t)$ can be decomposed into two contributions; one is a local contribution of the form $\left(\mu / 2 c_{s}\right)\left[v(x, t)-v_{0}\right]$, where $\mu$ is the linear elastic shear modulus and $c_{s}$ is the shear wave speed, and the other is a nonlocal (in space and time) contribution $s(x, t)$ [67-69]. This decomposition is valid for times shorter than $\mathcal{O}\left(H / c_{s}\right)$, before wave interaction with the boundaries is possible, and for these times the interfacial shear stress takes the form $[55,57,67-70]$

$$
\sigma_{x y}(x, y=0, t)=\tau_{d}-\frac{\mu}{2 c_{s}}\left[v(x, t)-v_{0}\right]+s(x, t) .
$$

In many studies available in the literature, the idealized infinite system limit $H \rightarrow \infty$ is considered, for which Eq. (3) is valid at all times. The term $\left(\mu / 2 c_{s}\right)\left[v(x, t)-v_{0}\right]$ physically represents wave radiation from the interface to the bodies that form it and is, therefore, known as the radiationdamping term [37-40]. It is associated with "damping" because, from the perspective of the interface, it acts as a viscous stress with $\mu / 2 c_{s}$ being the effective viscosity. This term makes an important contribution to stress drops in frictional rupture, as is shown next.

Consider a point along a frictional interface that is initially located ahead of a propagating frictional rupture and whose slip velocity is $v \approx 0$, which represents $v_{0}$ in Eq. (3). When the frictional rupture goes through this point, the stress and slip velocities vary significantly. Suppose then that the system height $H$ is sufficiently large such that the spatiotemporal integral decays once the rupture goes sufficiently far ahead, $s(x, t) \rightarrow 0$, but the radiation damping contribution is still valid (i.e., shear waves do not have enough time to propagate to the far boundaries and back). Under these conditions, the slip velocity $v_{\text {res }}$ at the spatial point under consideration, now far behind the frictional rupture, is determined by the shear stress balance $\sigma_{x y}=\tau$ and satisfies

$$
\tau_{\mathrm{ss}}\left(v_{\mathrm{res}}^{0}\right)+\frac{\mu}{2 c_{s}} v_{\mathrm{res}}^{0} \simeq \tau_{d},
$$

where $v_{\text {res }}^{0} \gg v_{0}$ and $s(x, t) \rightarrow 0$ are used. Note that the superscript " 0 " in $v_{\text {res }}^{0}$ represents the fact that it is the theoretically predicted residual slip velocity under the assumption that $s(x, t)=0$ far behind the propagating rupture front. The residual stress at this point takes the form $\tau_{\text {res }}=\tau_{d}-\left(\mu / 2 c_{s}\right) v_{\text {res }}^{0}$, and, consequently, a finite stress drop of magnitude

$$
\Delta \tau \simeq \frac{\mu}{2 c_{s}} v_{\mathrm{res}}^{0}
$$

is expected to emerge on times shorter than $\mathcal{O}\left(H / c_{s}\right)$.

A geometric representation of Eqs. (4) and (5) is shown in Fig. 2(b) for the $N$-shaped $\tau_{\mathrm{ss}}(v)$ and in Fig. 2(c) for the no-minimum $\tau_{\mathrm{ss}}(v)$, both shown in Fig. 2(a). In Figs. 2(b) and 2(c), the left-hand-side of Eq. (4) $\tau_{\mathrm{ss}}(v)+\left(\mu / 2 c_{s}\right) v$ is regarded as an effective steady-state curve and is plotted by a dashed line. The radiation-damping contribution $\left(\mu / 2 c_{s}\right) v$ in Fig. 2(b) shifts the location of the effective (finite-time) steady-state slip rate to lower rates [compared to the strict steady state represented by the rightmost intersection point in Fig. 2(a)]. In Fig. 2(c), it gives rise to an effective (finite-time) steady-state slip rate, which simply does not exist for the no-minimum curve in Fig. 2(a). This result shows that Eq. (5) is valid independently of the properties of $\tau_{\mathrm{ss}}(v)$. Note that relevant solutions $v_{\text {res }}^{0}$ to Eq. (4) exist only if $\tau_{d}$ is larger than the effective (finite-time) minimum of the steady-state friction curve, as is highlighted in Figs. 2(b) and 2(c). The very same condition plays a central role in the analysis of Ref. [39], where the conditions for rupture mode selection (selfhealing pulses versus cracklike) are extensively discussed. Somewhat related issues are also discussed in Ref. [71].

We note that, once $\Delta \tau$ is assumed to exist, the relation in Eq. (5) between $\Delta \tau$ and $v_{\text {res }}$ as given quantities is a known elastodynamic relation [72], which has previously received some experimental support (e.g., see Fig. 8 in Ref. [73]) and is strongly supported by recent experimental data we have extracted from recent experimental works [35,36]; see Sec. IV. To the best of our knowledge, however, none of these works (except for Ref. [39], as mentioned above) predict the residual slip velocity $v_{\text {res }}$ to emerge from the combined effect of the steady-state friction curve $\tau_{\mathrm{ss}}(v)$ and of the radiation-damping term $\left(\mu / 2 c_{s}\right) v$ as predicted in Eq. (4), and none of them interpret Eq. (5) as a major 
contribution to stress drops $\Delta \tau$ in frictional rupture. In the context of a quasistatic fault model of Ref. [74], the radiation-damping term $\left(\mu / 2 c_{s}\right) v$ is added in an ad hoc manner in order to avoid unbounded slip velocities from emerging during frictional instabilities in the quasistatic formulation. It is noted in this context [74] that the effective viscosity $\mu / 2 c_{s}$ affects the magnitude of the stress drop $\Delta \tau$ when $\tau_{\mathrm{ss}}(v)$ has no minimum [cf. Fig. 2(c)]; nevertheless, the general physical picture in which the radiation-damping term significantly contributes to stress drops in frictional rupture, independently of the properties of $\tau_{\mathrm{ss}}(v)$, has not been discussed.

When the radiation-damping term $\left(\mu / 2 c_{s}\right) v$ in Eq. (4) does not faithfully represent the physics of a given system, no well-defined finite stress drop is expected to accompany a rapid frictional rupture. This situation can happen in two generic cases; first, in the limit of thin bodies $H \rightarrow 0$, where essentially there is no bulk to radiate energy into, the radiation-damping term $\left(\mu / 2 c_{s}\right) v$ simply does not exist to begin with. In this case, frictional rupture exists, but it is not accompanied by any stress drop, as shown previously in Ref. [75] and here in Fig. 6. Second, the radiation-damping term $\left(\mu / 2 c_{s}\right) v$, which exists at relatively short times [shorter than $\mathcal{O}\left(H / c_{s}\right)$ ], is expected to vanish in the long time limit $t \gg H / c_{s}$. This limit can be probed by performing experiments or simulations with long enough systems for long enough times. Indeed, simulations of effectively long systems yield rupture fronts with no stress drop; see Fig. 6(a) in Ref. [76] and details therein. Finally, note that the radiation-damping term $\left(\mu / 2 c_{s}\right) v$ is expected to decrease to zero in discrete steps corresponding to each wave reflection from the system's boundaries. Experimental evidence for the stepwise nature of the decrease in the radiationdamping term (associated with discrete wave reflections) is discussed below.

\section{A. A perturbative approach for rapid rupture and the slow rupture limit}

The main theoretical prediction in Eqs. (4) and (5) is obtained under the assumption that the spatiotemporal integral term $s(x, t)$ in Eq. (3) vanishes well behind the propagating rupture. While this idealized assumption is physically sensible and is reasonably supported by simulational and experimental results for a rapid rupture discussed below, the long-range spatiotemporal nature of $s(x, t)$ may suggest that it does not strictly vanish in many realistic situations. Consequently, our goal here is to understand how $v_{\text {res }}^{0}$ and $\Delta \tau$ of Eqs. (4) and (5) change in the presence of a finite, yet small, $s(x, t)$.

To address this question, we denote the typical value of $s(x, t)$ at the tail of a rapidly propagating rupture front by $s$ and consider a perturbed solution of the form $v=v_{\text {res }}^{0}+$ $\delta v_{\text {res. }}$. We then expand Eq. (3), which in the present context takes the form $\Delta \tau(v)=\left(\mu / 2 c_{s}\right) v-s$, to linear order in $\delta v_{\text {res }}$ (it is already linear in $s$ ) to obtain

$$
\begin{aligned}
\frac{\delta v_{\mathrm{res}}}{v_{\mathrm{res}}^{0}} & =2 \frac{c_{s} s}{v_{\mathrm{res}}^{0}} \frac{s}{\mu}(1+\epsilon)^{-1}, \quad \epsilon \equiv 2 \frac{c_{s}}{v_{\mathrm{res}}^{0}} \frac{\sigma}{\mu} \frac{\partial f_{\mathrm{ss}}}{\partial \log \left(v_{\mathrm{res}}^{0}\right)}, \\
\Delta \tau+\delta(\Delta \tau) & =\frac{\mu}{2 c_{s}} v_{\text {res }}^{0}\left(1-\epsilon \frac{\delta v_{\text {res }}}{v_{\mathrm{res}}^{0}}\right) .
\end{aligned}
$$

Note that the internal state field $\phi$ is assumed above to be slaved to $v$ and that $f_{\mathrm{sS}}(v)=\tau_{\mathrm{ss}}(v) / \sigma$ is the steady-state friction coefficient.

Equation (6) reveals an interesting result; while both $v_{\text {res }}^{0}$ and $\Delta \tau$ attain corrections that are linear in $s$, as expected from a linear perturbation approach, the actual smallness of the corresponding corrections may be quite different due to the appearance of $\epsilon$. $\epsilon$ is a product of $c_{s} / v_{\text {res }}^{0} \gg 1, \sigma / \mu \ll 1$, and $\left|\left\{\partial f_{\text {ss }} /\left[\partial \log \left(v_{\text {res }}^{0}\right)\right]\right\}\right| \ll 1[5,45]$, where the latter two contributions are expected to dominate the first one, leading to $|\epsilon| \ll 1$. Consequently, while $\epsilon$ is expected to have a negligible effect on $\delta v_{\text {res }}$, due to the appearance of the factor $(1+\epsilon)^{-1}$, it implies that $\delta(\Delta \tau) / \Delta \tau$ is a factor of $\epsilon$ smaller than $\delta v_{\text {res }} / v_{\text {res. }}^{0}$. We thus expect the stress drop $\Delta \tau$ to be far less sensitive to finite values of $s(x, t)$ compared to the residual slip velocity $v_{\text {res. }}$. This difference is demonstrated below.

Equations (4)-(6) are derived under the assumption of vanishing or small spatiotemporal contribution $s(x, t)$, valid for physical situations in which a rapid rupture emerges. Yet, when rupture velocities are negligible compared to elastic wave speeds, i.e., when a slow rupture emerges [41-44], characteristic slip velocities $v$ are expected to be small such that the assumption behind Eqs. (4) and (5) may no longer be valid. In fact, for a sufficiently slow rupture, we expect the spatiotemporal integral term $s(x, t)$ in Eq. (3) to be significantly larger than $\left(\mu / 2 c_{s}\right) v(x, t)$ such that

$\frac{2 c_{s} \Delta \tau}{\mu v_{\text {res }}} \gg 1$ and $\quad \Delta \tau \simeq-s(t)$ for slow rupture,

where $s(t)$ is determined by

$s(t) \simeq \frac{\mu^{\prime}}{2 \pi} \int_{-\infty}^{\infty} \frac{\partial_{x^{\prime}} \delta\left(x^{\prime}, t\right)}{x^{\prime}-x} d x^{\prime}$ for sufficiently large $x$.

Here, $\delta(x, t)$ is the slip displacement $[\dot{\delta}(x, t)=v(x, t)]$, $\mu^{\prime}=\mu$ for antiplane shear, and $\mu^{\prime}=\mu /(1-\nu)$ for in-plane shear ( $\nu$ is Poisson's ratio). Equation (8) is the quasistatic limit of the fully inertial integral term $s(x, t)$ [77], which is expected to be valid for a slow rupture, where inertial effects are negligible.

It is important to note that $s(t)$ of Eq. (8), and, consequently, also the stress drop $\Delta \tau$, may feature a nontrivial dependence on the rupture size and may attain finite values for long times in practical applications. In fact, a simple self-consistent dimensional analysis in the limit of a large rupture in an infinite system indicates that $s$, and, hence, also $\Delta \tau$, inversely scales with the square root of the rupture size. Furthermore, note that, while the stress drop 
$\Delta \tau$ predicted for a slow rupture in Eq. (7) is not related to the radiation-damping term, it is still not a purely interfacial property, but rather it involves the long-range elasticity of the bodies surrounding the interface.

\section{B. Estimates of the spatiotemporal integral contribution to the stress drop}

In the previous subsection, we discuss the contribution of the long-range spatiotemporal term $s(x, t)$ to the stress drop, denoted there by $s$. In most of the discussion, excluding the last part concerning a slow rupture, $s$ is assumed to be small compared to the radiation-damping contribution. However, we do not provide quantitative estimates for the relative magnitude of the two contributions, which is shown to be quantified by the ratio $\delta v_{\text {res }} / v_{\text {res }}^{0}$ [cf. Eq. (6)]. In this subsection, we provide such estimates in some strongly dynamic limiting cases. The basic idea is that, since $s$ emerges from long-range linear elastodynamics, one can use some benchmark linear elastic fracture mechanics (LEFM) crack solutions [78], in which a finite stress drop $\Delta \tau$ is prescribed, to estimate its relative magnitude.

While the whole purpose of the present paper is to show that $\tau_{\text {res }}$ is not an a priori known interfacial quantity, and, hence, also $\Delta \tau=\tau_{d}-\tau_{\text {res }}$ is not a priori known, LEFM solutions are still useful in estimating the relative magnitude of $s$ through $\delta v_{\text {res }} / v_{\text {res }}^{0}$. The point is that one can use the prescribed $\Delta \tau$ to obtain $v_{\text {res }}^{0}$ according to Eq. (5) for $s=0$, then extract from the benchmark LEFM crack solution $v_{\text {res }}$ for $s \neq 0$, and finally obtain $\delta v_{\text {res }} / v_{\text {res }}^{0}=$ $\left(v_{\text {res }}-v_{\text {res }}^{0}\right) / v_{\text {res }}^{0}$ as an estimate for the relative magnitude of $s$ due to long-range linear elastodynamic interactions, which are properly captured by the crack solution.

To apply this procedure, we consider Broberg's selfsimilar crack solutions in infinite media [78]. In these solutions, two crack tips are assumed to symmetrically expand at a constant velocity $c_{r}$, starting from a zero crack length at $t=0$. Self-similarity, which significantly simplifies the problem, implies that all fields in the problem depend only on the dimensionless combination $x / c_{r} t$. While in general, as well as in the present work, frictional rupture propagation is not a self-similar process and the propagation speed is typically not constant, when the propagation speed approaches the limiting or asymptotic speed, self-similar conditions are reasonably approximated. Self-similar solutions are also used in a related analysis in Ref. [79] (cf. Appendix C3 therein), with the aim of deriving precise conditions for rupture mode selection (self-healing pulses versus cracklike), originally discussed in Ref. [39].

Applying these ideas to antiplane shear (mode-III symmetry) self-similar crack solutions [see Eq. (6.9.145) in Ref. [78]], we obtain in the limit $c_{r} \rightarrow c_{s}\left(c_{s}\right.$ is the limiting speed for mode III) $\delta v_{\text {res }} / v_{\text {res }}^{0}=2 / \pi-1 \approx-0.36$ (see Appendix G). This result, which is, in fact, quantitatively supported by numerical simulations in Sec. III, suggests that, while the relative magnitude of $s$ is not extremely small under strongly dynamic mode-III conditions, it is still reasonably well within the range of validity of the linear perturbation theory of Eq. (6). Repeating the same procedure for in-plane shear (modeII symmetry) self-similar crack solutions [see Eq. (6.9.85) in Ref. [78] ] in the limit $c_{r} \rightarrow c_{R}\left(c_{R}\right.$ is the Rayleigh wave speed, the limiting speed for mode II), we obtain that $\delta v_{\text {res }} / v_{\text {res }}^{0}$ varies between -0.613 and -0.417 (the exact value depends on Poisson's ratio); see Appendix G for details.

These latter estimates are quantitatively similar to the mode-III estimate in the corresponding limiting case, demonstrating that $|s|$ is smaller than the radiation-damping contribution for an in-plane frictional rupture under strongly dynamic conditions. In both cases, the linear perturbation theory of Eq. (6) seems to be reasonably valid. We note that, while the estimates above are based on bilateral (self-similar) rupture propagation, similar results are expected to emerge for unilateral propagation. Finally, we stress again that the influence of $s$ on $\Delta \tau$ through $\delta v_{\text {res }} / v_{\text {res }}^{0}$ in Eq. (6) is strongly reduced by the small dimensionless factor $\epsilon$ (defined there). This discussion concludes the presentation of our main theoretical predictions, encapsulated in Eqs. (4)-(8). In the next sections, we provide simulational and experimental support to these predictions.

\section{SIMULATIONAL SUPPORT}

At this point, we first set out to test the predictions in Eqs. (4)-(8) against extensive numerical simulations. To that aim, we consider two semi-infinite bodies in frictional contact. The advantage of considering infinite-height bodies, i.e., the $H \rightarrow \infty$ limit, is that the interfacial relation in Eq. (3) becomes exact at all times, unlike for finite bodies. We also employ periodic boundary conditions, with periodicity $W$, in the sliding direction. We perform spectral boundary integral method [67-69] calculations under antiplane shear (mode-III symmetry) deformation conditions, which are similar to-yet somewhat simpler than-the inplane shear (mode-II symmetry) deformation conditions considered up to now [8]. The main simplification is that the displacement field in the mode-III problem $\boldsymbol{u}(x, y, t)=$ $u_{z}(x, y, t) \hat{z}$ (the unit vectors satisfy $\left.\hat{z} \perp \hat{\boldsymbol{x}}, \hat{\boldsymbol{y}}\right)$ is essentially scalar. The basic field $u_{z}(x, y, t)$ satisfies the bulk elastodynamic equation $\mu \nabla^{2} u_{z}=\rho \ddot{u}_{z}$, together with $v(x, t) \equiv \dot{u}_{z}\left(x, y=0^{+}, t\right)-\dot{u}_{z}\left(x, y=0^{-}, t\right)$ and $\tau(x, t) \equiv$ $\sigma_{y z}(x, y=0, t)=\mu \partial_{y} u_{z}(x, y=0, t)$. Equation (3) remains valid, where $\sigma_{x y}(x, y=0, t)$ is replaced by $\sigma_{y z}(x, y=0, t)$ and the integral term $s(x, t)$ corresponds to mode III; see Appendix A for more details.

The employed interfacial constitutive law features the generic properties discussed above, with $f(|v|, \phi)$ of 
Eq. (1) that reduces under steady-state conditions to either the $N$-shaped or the no-minimum curves of Fig. 2(a) [the exact expressions for $f(|v|, \phi)$ can be found in Appendix B] and with $g(\cdot)$ of Eq. (2) that is given by $g=1-|v| \phi / D[5,45,46,49,52]$. The bodies are loaded by a constant driving stress $\tau_{d}$, as depicted schematically in Fig. 2(a), and the frictional rupture is nucleated by introducing Gaussian perturbations of the proper amplitude into a homogeneous state of very low slip velocity $v_{0}$ [that corresponds to the leftmost intersection point in Fig. 2(a)], following the theoretical framework of Ref. [80] (the details of the nucleation procedure are described in Appendices).

An example of the stress distribution of an emerging frictional rupture is shown in Fig. 3(a). The figure reveals two rapid rupture fronts propagating in opposite directions (at $84 \%$ of the shear wave speed $c_{s}$ ), where the stress ahead of the two fronts is the applied stress $\tau_{d}$. As the emerging rupture is rapid, i.e., propagating at a speed comparable to the elastic wave speed, the relevant prediction is given by Eq. (5). As predicted, the observed stress left behind the two rapid fronts, $\tau_{\text {res }}$, is constant and smaller than the driving stress $\tau_{d}$, giving rise to a finite stress drop $\Delta \tau$. Following the discussion above, since in these calculations $H \rightarrow \infty$, the finite stress drop persists indefinitely [while in finite size systems it persists for times $\sim \mathcal{O}\left(H / c_{s}\right)$; cf. the experiments of Ref. [35], discussed later]. The stress drop $\Delta \tau$ observed in Fig. 3(a) quantitatively agrees with the prediction in Eq. (5), as stated in the figure legend.

In Fig. 3(b), we present the slip-velocity distribution that corresponds to the snapshot shown in Fig. 3(a). As predicted, the slip velocity attains a plateau level $v_{\text {res }}$ behind the propagating rupture fronts, and the residual velocity $v_{\text {res }}$ is used to normalize the slip-velocity distribution. The simulationally measured residual velocity $v_{\text {res }}$ deviates by approximately $35 \%$ from the theoretical prediction in Eq. (5), in quantitative agreement with the estimate provided in Sec. II B for the spatiotemporal integral contribution to the stress drop in this case (see also Appendix G). Consequently, while the upper equation in Eq. (6) implies that $s$ is, in fact, not very small, the prediction for $\Delta \tau$ works perfectly fine due to the $\epsilon \ll 1$ factor in the lower equation in Eq. (6).

In order to quantitatively test the latter prediction for a rapid rupture over a range of physical conditions, we first solve Eq. (4) to obtain $v_{\text {res }}^{0}\left(\tau_{d}\right)$ and then plug it into Eq. (5) to obtain $\Delta \tau\left(\tau_{d}\right)$, where the latter is plotted in solid lines in Fig. 3(c) [the two solid lines correspond to both the $N$-shaped or the no-minimum steady-state friction laws shown in Fig. 2(a)]. We then numerically calculate $\Delta \tau$ [as demonstrated in Fig. 3(a)] for various driving stresses $\tau_{d}$, for both the $N$-shaped or the no-minimum steady-state friction curves shown in Fig. 2(a). The numerical results (discrete symbols) are superimposed on the theoretical prediction in Fig. 3(c) [the lowest numerical data point on the lower curve corresponds to Figs. 3(a) and 3(b)].
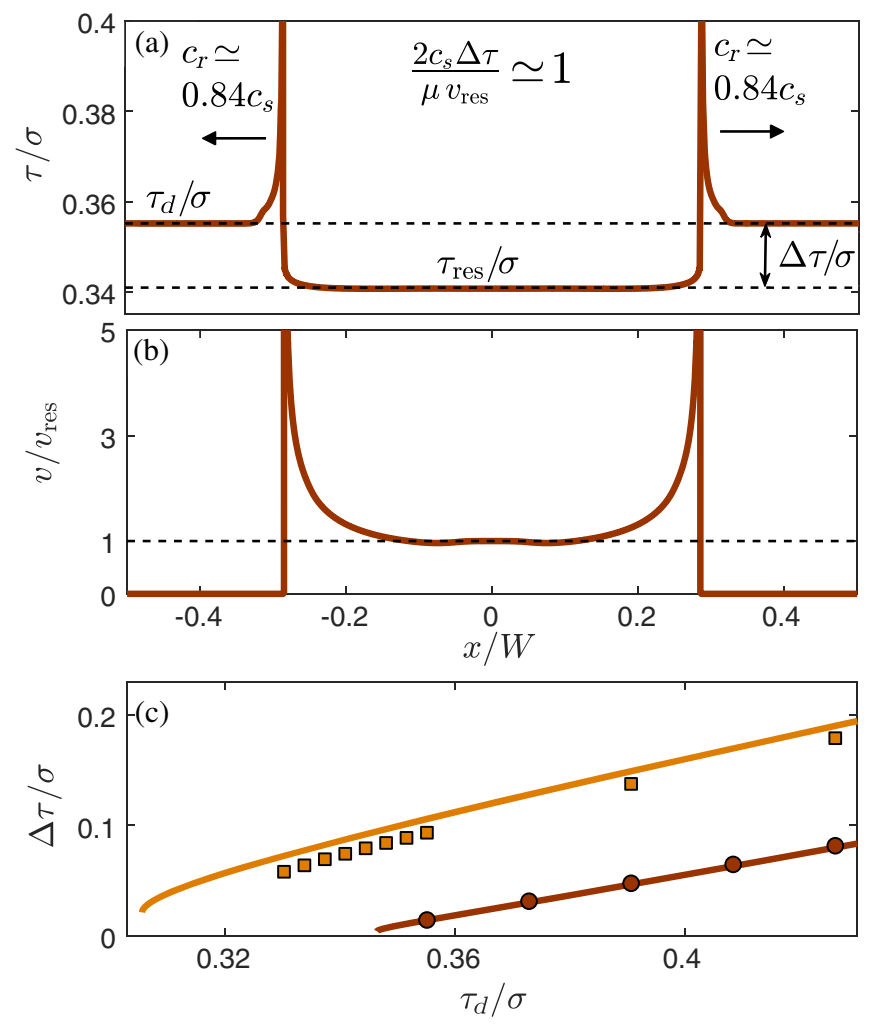

FIG. 3. (a) A snapshot of the frictional stress $\tau(x)$ (normalized by $\sigma$ ) during rupture propagation that emerges in dynamic simulations with the $N$-shaped steady-state friction law of Fig. 2(a) and $\tau_{d}=0.355 \sigma$ (see the text for details). The snapshot reveals two rapid rupture fronts propagating at an instantaneous speed $c_{r} \simeq 0.84 c_{s}$ in opposite directions into regions characterized by the applied stress $\tau_{d}$ and leaving behind them a welldefined residual stress $\tau_{\text {res }}<\tau_{d}$. Consequently, a well-defined and finite stress drop $\Delta \tau$ emerges. Note that the $y$ axis is truncated at $\tau / \sigma=0.4$ for visual clarity (the maximal value of $\tau / \sigma$ is 0.58 ) and that $x$ is normalized by the system length $W$ [the $x$ axis is shared with (b)]. (b) The slip velocity $v(x)$ that corresponds to the snapshot shown in (a), normalized by the simulationally measured residual velocity $v_{\text {res }}$ (see the text for a discussion). The $y$ axis is also truncated for visual clarity, and $x$ is normalized by the system length $W$. (c) The theoretical predictions of Eqs. (4) and (5) for $\Delta \tau\left(\tau_{d}\right)$ of a rapid rupture (solid lines), both for the $N$-shaped steady-state friction law of Fig. 2(a) (solid brown line, lower curve) and for the no-minimum law of Fig. 2(a) (solid orange line, upper curve). As expected [cf. Figs. 2(b) and 2(c)], the former is smaller than the latter. The corresponding numerical results, obtained from the spatial stress distribution of a frictional rupture such as the one shown in (a), are shown by the discrete symbols [circles for the $N$-shaped law, where the leftmost data point corresponds to the results shown in (a), and squares for the no-minimum law]. See the text for additional discussion.

The agreement between the theoretical prediction and the numerical results for $\Delta \tau$ is very good, where it is better for the $N$-shaped steady-state friction law (lower curve) than for the no-minimum steady-state friction law (upper curve). This difference is fully accounted for by the magnitude and 


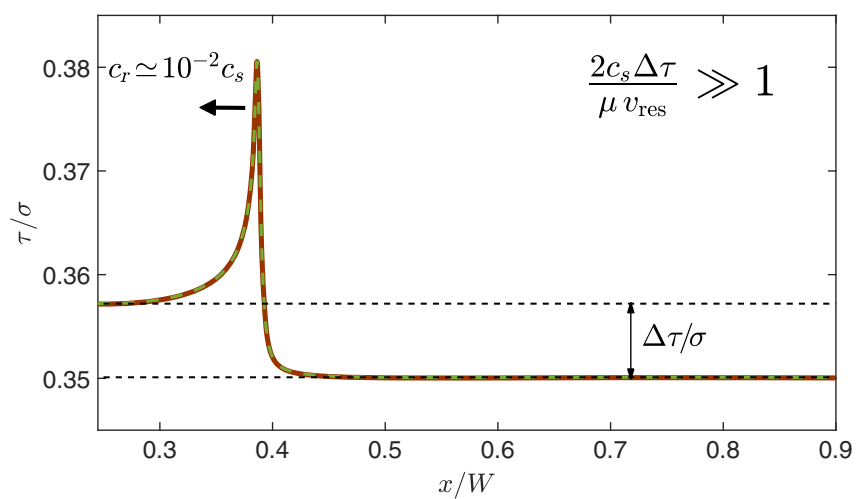

FIG. 4. A snapshot of the frictional stress $\tau(x)$ (normalized by $\sigma, x$ is normalized by the system length $W$ ) of a slow rupture (solid red line) propagating at about $1 \%$ of the shear wave speed $c_{s}$ and leaving behind it a stress drop $\Delta \tau$ (see Appendix E for details; note that the other rupture edge is not shown). The stress drop satisfies $\left(2 c_{s} \Delta \tau / \mu v_{\text {res }}\right) \gg 1$, as stated in the legend and in agreement with Eq. (7). $\tau_{d}+s(x, t)$ (dashed green line), where $s(x, t)$ is given by the quasistatic integral of Eq. (8), is superimposed. See the text for additional discussion.

sign of $\epsilon$, directly affected by the $\left\{\partial f_{\mathrm{ss}} /\left[\partial \log \left(v_{\mathrm{res}}^{0}\right)\right]\right\}$ term [cf. Eq. (6)].

The contribution of the spatiotemporal integral $s(x, t)$, which is smaller than the radiation-damping contribution for a rapid rupture, may be a dominant effect for a slow rupture, as discussed around Eqs. (7) and (8). To test this possibility, we generate a slow rupture by changing the frictional parameters, the loading level, and the nucleation procedure, as explained in Appendix E. An example of such a slow rupture is shown in Fig. 4 (solid red line), exhibiting a rupture front propagating at a velocity 2 orders of magnitude smaller than $c_{s}$ and leaving behind it a stress drop $\Delta \tau$. We first verify that $\left(2 c_{s} \Delta \tau / \mu v_{\text {res }}\right) \gg 1$, as predicted by Eq. (7) (and as stated in the figure legend). To test whether indeed $\Delta \tau \simeq-s(t)$ [cf. Eq. (7)], we use the slip displacement gradient $\partial_{x} \delta(x, t)$ obtained from the dynamical simulation to calculate the quasistatic integral in Eq. (8) for any point $x$ along the interface at $t$ corresponding to the snapshot in Fig. 4. The result for $\tau_{d}+$ $s(x, t)$ is superimposed on $\tau(x, t)$ in Fig. 4 (dashed green line), demonstrating excellent agreement with the fully dynamic result and, in particular, validating $\Delta \tau \simeq-s(t)$ for this $t$. Consequently, our simulations strongly support the theoretical predictions in Eqs. (4)-(8), for both a rapid and a slow frictional rupture. Next, we discuss direct experimental support for these predictions.

\section{EXPERIMENTAL SUPPORT}

In the previous section, we provide strong simulational support to the theoretical predictions in Eqs. (4)-(8). Our goal here is to test these predictions against experimental data. Equation (4) predicts the slip velocity $v_{\text {res }}$ behind the frictional rupture once the steady-state friction curve $\tau_{\mathrm{ss}}(v)$ is known. The latter is not always known a priori over a sufficiently wide range of steady-state slip velocities. In fact, measuring both the frictional stress $\tau_{\text {res }}$ and the slip velocity $v_{\text {res }}$ behind rupture fronts allows one to extract $\tau_{\text {ss }}(v)$. In this case, any triplet $\left(\tau_{d}, \tau_{\text {res }}, v_{\text {res }}\right)$ is predicted to follow either Eq. (5) for a rapid rupture or Eq. (7) for a slow rupture, independently of the steady-state friction law $\tau_{\mathrm{ss}}(v)$.

Measurements of both $\tau_{\text {res }}$ and $v_{\text {res }}$ behind rupture fronts for various $\tau_{d}$ have been recently performed by two independent experimental groups using two different experimental systems and techniques [35,36]. The first focuses on the frictional dynamics along the interface between two blocks of Homalite probed through a novel ultrahigh full-field imaging technique [35]. The second focuses on the frictional dynamics along the interface between two blocks of poly(methylmethacrylate) probed through a combination of high-speed interfacial imaging (via a method of total internal reflection) and simultaneous measurements of the deformation fields slightly above the interface [36]. Here, we use the data reported in these works to quantitatively test our predictions.

We start with Ref. [35], where the observed rupture fronts are all in the rapid (fully inertial) regime, and, hence, the relevant prediction is given in Eq. (5). To test this prediction, we extract the relevant experimental data from Ref. [35] and plot in Fig. 5 the resulting $\Delta \tau$ versus $v_{\text {res }}$ against the linear $\Delta \tau\left(v_{\text {res }}\right)$ relation of Eq. (5), with a slope that corresponds to $\mu / 2 c_{s}$ of Homalite (see the figure caption for additional details). The results reveal excellent agreement between the experimental data and the theoretical prediction, without any free parameter. The theoretical relation in Eq. (5) predicts $v_{\text {res }}^{0}$ by assuming that the longrange spatiotemporal contribution $s(x, t)$ is negligibly small, while in the experimental data we just use the measured $v_{\text {res }}$. The fact that there are no significant horizontal deviations of the experimental data from the theoretical line of Eq. (5), i.e., that $v_{\text {res }}$ is close to $v_{\text {res}}^{0}$, indicates that $s(x, t)$ is smaller in these experiments compared to the estimate provided in Sec. II B. The origin of the latter discrepancy is not yet clear.

The experimental data included in Fig. 5 are obtained in the short time regime, before any wave reflection from the system's boundaries, for which Eqs. (4) and (5) are valid. Yet, for a single case, measurements are reported after the first wave reflection (but before the second one); cf. Fig. 4(c) in Ref. [35]. Under these conditions, we expect the ordinary radiation-damping term $\left(\mu / 2 c_{s}\right) v$ to be replaced by a smaller term. Consequently, Eq. (4) predicts that $v_{\text {res }}$ should increase, exactly as is experimentally observed in Fig. 4(c) in Ref. [35] (denoted as a "reflected rupture" in the figure), thus providing direct evidence for the stepwise reduction of the radiation-damping term with discrete wave reflections.

We now turn to the experiments of Ref. [36], which report on extensive measurements of $\tau_{\text {res }}$ and $v_{\text {res }}$; cf. Fig. 3(a) there. 


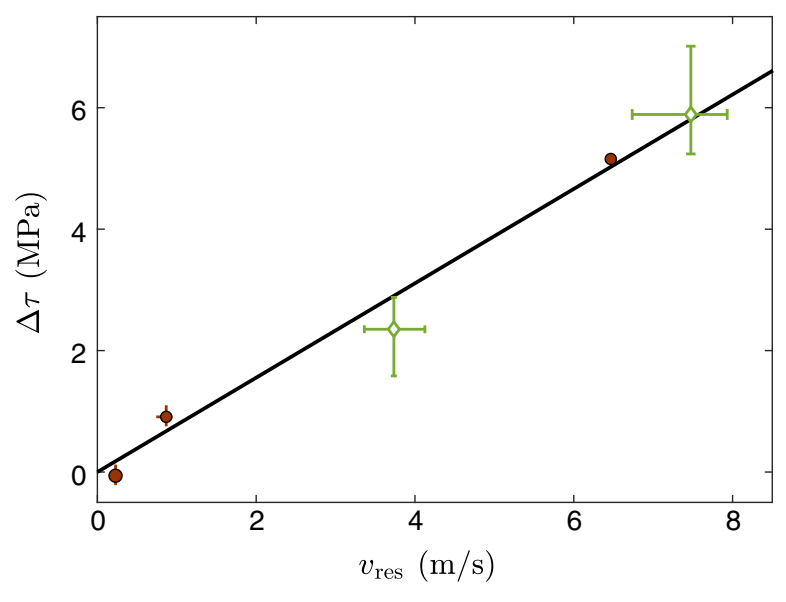

FIG. 5. The theoretical prediction of Eq. (5) is plotted for Homalite by the solid line, with the following high strain rate material parameters $\mu=1.96 \mathrm{GPa}$ and $c_{s}=1263 \mathrm{~m} / \mathrm{s}$ [35,81], which uniquely determine the slope of the stress drop $\Delta \tau$ versus the slip rate $v_{\text {res }}$ behind a rupture front. Experiments on the rupture of frictional interfaces composed of two blocks of Homalite are performed in Ref. [35], where a novel ultrahigh full-field imaging technique is employed to directly measure $\tau_{\text {res }}$ and $v_{\text {res }}$ behind rupture fronts. Experiments for different values of the applied shear stress $\tau_{d}$ are performed, which allow us to extract from Fig. 8(a) of Ref. [35] five triplets $\left(\tau_{d}, \tau_{\text {res }}, v_{\text {res }}\right)$ (see details in Appendix F). For each triplet, we calculate $\Delta \tau \equiv \tau_{d}-$ $\tau_{\text {res }}$ and then superimpose the resulting $\Delta \tau$ versus $v_{\text {res }}$ on the theoretical prediction (discrete symbols). The symbols (and colors) differentiate data obtained from high-resolution (full brown circles) and low-resolution (empty green diamonds) measurements, following the classification of Ref. [35]. The symbol size and/or error bar represent the full range of measurements reported on in Fig. 8(a) of Ref. [35] per imposed farfield conditions.

In order to test our predictions, we need also measurements of the driving stress $\tau_{d}$, which allow us to extract the stress drop $\Delta \tau=\tau_{d}-\tau_{\text {res. }}$. The corresponding extensive measurements of $\tau_{d}$ are not presented in Ref. [36], though $\tau_{d}$ is presented for two highly relevant examples in Fig. 2(b) there (where $\tau_{d}$ is denoted by $\tau_{0}$ ). One example corresponds to a very rapid rupture (in fact, it is a supershear rupture, propagating at nearly the dilatational wave speed) and the other to a slow rupture (propagating at about $10 \%$ of the Rayleigh wave speed). These data are exactly what is needed in order to test our predictions, in particular, regarding the change in behavior for a slow rupture. For a rapid rupture, we extract from Fig. 2(b) of Ref. [36] (blue data) $\Delta \tau=1.17 \mathrm{MPa}$ and from Fig. 1(d) (bottom) $v_{\text {res }} \simeq 1.5 \mathrm{~m} / \mathrm{s}$ (see additional details in Appendix F). Using the reported values $\rho=1170 \mathrm{~kg} / \mathrm{m}^{3}$ and $c_{s}=1345 \mathrm{~m} / \mathrm{s}$ [36], together with $\mu=\rho c_{s}^{2}$, we obtain $\left(2 c_{s} \Delta \tau / \mu v_{\text {res }}\right)=0.99$. This result is in great quantitative agreement with the prediction for a rapid rupture in Eq. (5) and is fully consistent with the independent experimental data presented in Fig. 5. This result indicates that the long-range spatiotemporal contribution $s(x, t)$ is indeed small also in the experiments of Ref. [36].

We then extract the corresponding data for the slow rupture, obtaining from Fig. 2(b) of Ref. [36] (red data) $\Delta \tau=0.242 \mathrm{MPa}$ and from Fig. 1(d) (top) $v_{\text {res }} \simeq 0.02 \mathrm{~m} / \mathrm{s}$. Using these data, we obtain $\left(2 c_{s} \Delta \tau / \mu v_{\text {res }}\right) \simeq 15 \gg 1$, in agreement with the theoretical prediction for a slow rupture in Eq. (7). The results presented in the past two sections provide strong simulational and experimental support for our theoretical predictions and, hence, for the proposed picture of the physical origin of stress drops in frictional rupture. As stress drops are important for the possible emergence of cracklike behavior in frictional rupture, future studies of the latter should be based on the results presented in this paper.

\section{DISCUSSION AND CONCLUDING REMARKS}

The possible deep relations between frictional rupture and ordinary fracture provide a powerful conceptual and quantitative framework to understand frictional dynamics in a wide variety of physical contexts. This framework is extensively used to interpret and quantify geophysical observations [26,28], as well as a broad spectrum of laboratory phenomena [29-35]. For example, a recent series of careful laboratory experiments [32-34] demonstrated that, when the analogy between frictional rupture and ordinary fracture holds, the dynamic propagation of laboratory earthquakes and their arrest can be quantitatively understood to an unprecedented degree [25]. Yet, the fundamental physical origin and range of validity of the analogy between frictional rupture and ordinary fracture are not yet fully understood. In this paper, we developed a comprehensive and fundamental understanding of why, how, and to what extent frictional rupture might be viewed as an ordinary fracture process.

An important ingredient in the analogy is the emergence of a finite and well-defined stress drop $\Delta \tau=\tau_{d}-\tau_{\text {res }}$, the difference between the applied driving stress $\tau_{d}$ and the residual stress $\tau_{\text {res }}$, in frictional rupture. In the first part of the paper, we showed that, contrary to widely adopted assumptions, the residual stress $\tau_{\text {res }}$ is not a characteristic property of frictional interfaces. Rather, for a rapid rupture, $\tau_{\text {res }}$ is shown to crucially depend on elastodynamic bulk effects - mainly wave radiation from the frictional interface to the bodies surrounding it but also long-range elastodynamic bulk interactions [encapsulated in the integral term $s(x, t)$ in Eq. (3)] — and the applied driving stress $\tau_{d}$ itself, in addition to the contribution of the slip-rate dependence of the constitutive friction law. Notably, we showed that for a rapid rupture the deviation of $\tau_{\text {res }}$ from $\tau_{d}$, i.e., the existence of a finite stress drop $\Delta \tau$, is a finite-time effect, mainly limited by the wave travel time in finite systems. For a slow rupture, it is shown that, if a stress drop exists, it is intimately related to the long-range quasistatic elasticity of the bodies surrounding the interface, again not exclusively 
to interfacial physics. Our theoretical predictions are supported by extensive computations and existing experimental data from two independent laboratory experiments.

Our findings have important implications that go beyond their basic nature; first, the results show that the widely used slip-weakening models $[9,10]$, in which the existence of a residual stress $\tau_{\text {res }}<\tau_{d}$ is a priori assumed (as a fixed interfacial property), should be employed with care. In particular, as $\tau_{\text {res }}$ has been shown to depend on the externally applied stress $\tau_{d}$, on the properties of the bodies surrounding the interface, and on the rate dependence of the frictional constitutive behavior, $\tau_{\text {res }}$ cannot be assumed to be fixed. Rather, it should be self-consistently calculated from the coupled interface-bulk problem. Somewhat related conclusions in relation to slip-weakening models, based on measurements of evolving local friction during spontaneously developing laboratory earthquakes, have been drawn in Ref. [35]. We also note that the dependence of $\tau_{\text {res }}$ on the bulk constitutive relation and on long-range bulk-mediated interactions may give rise to interesting effects for more complicated bulk constitutive relations (e.g., viscoelasticity) and in the presence of repeated rupture events [82]. Such effects should be explored in future work.

The existence of a finite stress drop $\Delta \tau$ generically leads to accelerating frictional rupture under stress-controlled farfield loading conditions $\tau_{d}$ if $\Delta \tau$ is independent of the rupture size $L$. In these situations, an inertia-limited rapid rupture is expected to emerge on timescales for which wave interaction with the outer boundaries does not exist, and $\Delta \tau$ is controlled by elastodynamic bulk effects. These conditions are typically realized in many geological and laboratory earthquakes [26,28-35]. On the other hand, slow rupture propagation - a widely observed, yet highly debated and elusive phenomenon [41-44] — is expected to feature smaller stress drops $\Delta \tau$ that may decrease with increasing rupture size $L$, such that rupture acceleration is limited.

The possible $L$ dependence of $\Delta \tau$ and its possible relations to the emergence of a slow rupture should be further explored in the future. In addition, as the whole discussion in this paper is valid for stress-controlled farfield loading conditions (characterized by $\tau_{d}$ ), future work should also consider velocity-controlled far-field loading conditions, where finite stress drops might emerge from different physical considerations [83]. Finally, as the existence of a finite stress drop $\Delta \tau$ does not in itself guarantee the cracklike behavior of frictional rupture, future work should clarify to what extent the analogy to ordinary cracks can, in fact, quantitatively account for the dynamics of frictional rupture. All in all, as stress drops are key quantities in frictional failure dynamics, we expect our results to provide a conceptual and quantitative framework to address various fundamental and applied problems in relation to the rupture dynamics of frictional interfaces, with implications for both laboratory and geophysical-scale phenomena.

\section{ACKNOWLEDGMENTS}

E. B. and J.-F.M. acknowledge support from the Rothschild Caesarea Foundation. E. B. acknowledges support from the Israel Science Foundation (Grant No. 295/ 16). J.-F. M., F. B., and T. R. acknowledge support from the Swiss National Science Foundation (Grant No. 162569). This research is made possible in part by the historic generosity of the Harold Perlman family. We are grateful to Ilya Svetlizky for pointing out the differences between rapid and slow rupture in the context of our work and the relevance of the experimental data of Ref. [36] to it. His comment has stimulated the discussion of slow rupture in this paper. In addition, we are grateful to him for pushing us to look at Broberg's self-similar solutions, eventually leading to the extraction of the estimates presented in Sec. II B.

\section{APPENDIX A: THE MAIN NUMERICAL METHOD}

The simulations discussed above rely on a spectral boundary integral formulation of the elastodynamic equations [67-69]. The latter relates the traction stresses acting along the interface between two linearly elastic half-spaces and the resulting displacements. For the mode-III (antiplane shear) elastodynamic problem studied in the manuscript, the interface is initially uniformly prestressed by $\tau_{d}$ and is set to slide at an extremely small steady velocity $v_{0}$, such that the shear tractions at the interface take the form

$$
\tau(x, t)=\tau_{d}-\frac{\mu}{2 c_{s}}\left[v(x, t)-v_{0}\right]+s(x, t) .
$$

The second right-hand-side term represents the instantaneous response to changes in the sliding velocity, the so-called radiation-damping term. As discussed in the manuscript, this term can be understood as the damping of interfacial energy due to elastic waves radiated into the infinite domain. The third term $s(x, t)$ accounts for the history and spatial distribution of interfacial displacements $u(x, t)$. Both $s(x, t)$ and $u(x, t)$ are related in the spectral domain via a convolution integral, whose expression can be found in Ref. [69]. Because of the spectral nature of the formulation, the simulated domain is taken to be periodic in the lateral direction, with periodicity $W$. The latter is chosen to be large enough to prevent any effect of the periodicity on the results reported in the manuscript.

A rupture is nucleated at the center of the domain by introducing a Gaussian perturbation of the slip velocity into an initial steady sliding state at $v_{0}$. The sliding velocity is then computed by combining Eq. (A1) and the rate and state-dependent friction law $\tau=\sigma \operatorname{sgn}(v) f(|v|, \phi)$ (see Appendix B for more details). $u(x, t)$ is then integrated in time using an explicit time-stepping scheme 


$$
u(x, t+\Delta t)=u(x, t)+0.5 v(x, t) \Delta t .
$$

Note that the factor 0.5 on the right-hand side of Eq. (A2) ensures that $v(x, t)$ is indeed the slip velocity. In order to guarantee the stability and the convergence of the numerical scheme, $\Delta t$ is defined as the time needed for a shear wave to travel a fraction 0.2 of one grid spacing, i.e., $\Delta t=0.2 \Delta x / c_{s}$. Additional information about the numerical scheme and the nucleation procedure can be found in Ref. [80], together with videos of similar rupture events.

\section{APPENDIX B: THE FRICTION LAWS}

The friction laws used in this work, and whose steadystate behaviors are plotted in Fig. 2, are related to the one used previously in Refs. $[58,80]$. A friction law is defined by the relation between the shear stress $\tau \equiv \sigma_{x y}$ and the compressive normal stress $\sigma \equiv-\sigma_{y y}$ at the interface, $\tau=\sigma \operatorname{sgn}(v) f(|v|, \phi)$, and by the evolution equation for state variable $\phi, \dot{\phi}=g(|v|, \phi)$. The constitutive functions $f(|v|, \phi)$ and $g(|v|, \phi)$ used in this work take the form

$$
\begin{aligned}
f(|v|, \phi)= & {\left[1+b \log \left(1+\frac{\phi}{\phi_{*}}\right)\right] } \\
& \times\left[\frac{f_{0}}{\sqrt{1+\left(v_{*} / v\right)^{2}}}+a \log \left(1+\frac{|v|}{v_{*}}\right)\right], \\
& g(|v|, \phi)=1-\frac{|v| \phi}{D} \sqrt{1+\left(v_{*} / v\right)^{2}},
\end{aligned}
$$

where $\phi$ represents the typical age or maturity of contact asperities that compose the interface at a microscopic scale [45]. In Eq. (B1), $f_{0}$ sets the scale of the dimensionless frictional resistance (friction coefficient), $b$ is the aging coefficient, and $a$ is related to the thermally activated rheology of contact asperities [45]. The function $\sqrt{1+\left(v_{*} / v\right)^{2}}$ that appears also in $g(|v|, \phi)$ ensures that, for vanishingly small steady-state velocities, $\phi$ saturates after extremely long times to a finite value of $D / v_{*}$ rather than diverges. As discussed in Ref. [80], this regularization makes no significant difference in the results discussed above. For the sake of notational simplicity, the regularization is hence omitted in the main text (though it is included in the calculations). Equations (B1) and (B2) lead to the steady-state friction curve in Fig. 2 with a minimum at an intermediate $v$ (brown solid line), while the nominimum steady-state friction curve (dash-dotted orange line) is obtained after neglecting the " +1 " in the $b$ term.

The reader is referred to Refs. $[58,80]$ for additional discussions about the formulations of Eqs. (B1) and (B2), which go beyond the conventional rate-and-state friction laws. Nevertheless, the results and conclusions discussed above are independent of the choice of the rate-and-state formulation.

\section{APPENDIX C: 1D RUPTURE FRONTS}

In this Appendix, we describe propagating steady-state rupture fronts in thin (quasi-1D) systems, where no stress drop emerges. We consider two long and thin linear elastic bodies of height $H$ in frictional contact, such that the momentum balance equation $\rho \ddot{\boldsymbol{u}}=\nabla \cdot \boldsymbol{\sigma}$ reduces to $[75,80,84]$

$H \bar{\mu}\left(c_{1 \mathrm{D}}^{-2} \partial_{t t}-\partial_{x x}\right) u(x, t)=\tau_{d}-\tau[v(x, t), \phi(x, t)]$,

where $u \equiv u_{x}, \bar{\mu}$ and $c_{1 \mathrm{D}}$ are the effective shear modulus and wave speed $[75,84]$, respectively, and $\tau_{d}$ is a constant driving stress (see Fig. 2).

Propagating 1D steady-state solutions then satisfy [80]

$$
\begin{gathered}
\bar{\mu} H c_{1 \mathrm{D}}^{-1}\left(1-\beta^{2}\right) \beta^{-1} v^{\prime}(\xi)=\tau_{d}-\tau[v(\xi), \phi(\xi)], \\
\beta c_{1 \mathrm{D}} \phi^{\prime}(\xi)=\phi(\xi) v(\xi) / D-1,
\end{gathered}
$$

where we define a comoving coordinate $\xi \equiv x-\beta c_{1 \mathrm{D}} t$, integrate out $u$, and eliminate partial time derivatives.

Steady-state rupture propagation is a dynamical process in which a homogeneous $V$ state invades a homogeneous $v_{0} \ll V$ state $[32,35,75,80]$, both shown in Fig. 2 as the intersections of the velocity-strengthening branches of the friction law with the driving stress $\tau_{d}$. We find these solutions for the friction law described in Sec. V, using a shooting method [85] (similar to that used in Refs. $[75,84]$ ). The solution is shown in Fig. 6. To normalize the stress fields, we use the definition $\tau_{m}$ as the maximal value $\tau$ attains in the profile, and $\ell \equiv\left[\left(1-\beta^{2} /\right) \beta\right]\left\{H V \bar{\mu} /\left[c_{1 \mathrm{D}}\left(\tau_{m}-\tau_{d}\right)\right]\right\}$ is the length scale over which the fields change, which can be calculated by a scaling analysis of Eq. (C2).

As seen in Fig. 6, the stress both ahead and behind the rupture front equals $\tau_{d}$; i.e., there exists no stress drop. A corollary is that no singularity is observed in Fig. 6 [compare to Figs. 3(a) and 4].

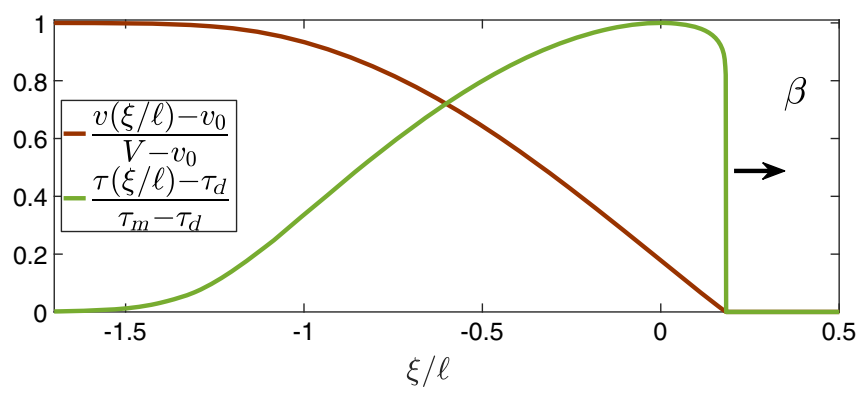

FIG. 6. The normalized spatial profiles of $\tau(\xi)$ and $v(\xi)$ near a steady-state rupture front edge propagating from left to right with a velocity $c_{r}^{1 \mathrm{D}}=\beta c_{s}$, with $\beta=0.144$ (see the text for details on the employed normalization). Note also that $\tau_{d} / \sigma=0.355$, exactly as in Fig. 2, though in the latter 2D case a rupture front with $\beta=0.84$ emerges [cf. Fig. 3(a)]. 
TABLE I. Values for all parameters used in MKS (MeterKilogram-Second system).

\begin{tabular}{lcr}
\hline \hline Parameter & Value & Units \\
\hline$\mu, \bar{\mu}$ & $9 \times 10^{9}$ & $\mathrm{~Pa}$ \\
$\sigma$ & $10^{6}$ & $\mathrm{~Pa}$ \\
$c_{s}, c_{1 \mathrm{D}}$ & 2739 & $\mathrm{~m} / \mathrm{s}$ \\
$D$ & $5 \times 10^{-7}$ & $\mathrm{~m}$ \\
$b$ & 0.075 & $\cdots$ \\
$v_{*}$ & $10^{-7}$ & $\mathrm{~m} / \mathrm{s}$ \\
$f_{0}$ & 0.28 & $\cdots$ \\
$\phi_{*}$ & $3.3 \times 10^{-4}$ & $\mathrm{~s}$ \\
$a$ & 0.005 & $\cdots$ \\
$H$ & 0.2 & $\mathrm{~mm}$ \\
\hline \hline
\end{tabular}

\section{APPENDIX D: PARAMETERS}

The parameters used for all the calculations described in this work, except for the slow rupture discussed in Appendix E, are given in Table I. Note that the values of the listed parameters are characteristic of some laboratory experiments (see Ref. [59] for details). However, the generic properties of the derived results are independent of the exact numbers and are relevant to a broad range of materials and physical situations. For example, $v_{*}$ that controls the velocity scale below which the system is in the stick phase can be taken to be significantly smaller.

\section{APPENDIX E: 2D SLOW RUPTURE FRONTS}

The very same constitutive framework can give rise to slow rupture fronts $\left(c_{r} \ll c_{s}\right)$, as demonstrated in Fig. 4. While the emergence of a slow rupture is of great interest, in general, in the present context we are interested in just generating a slow rupture and studying its properties in relation to the theoretical prediction in Eqs. (7) and (8). One way to generate a slow rupture within our constitutive framework is to use friction parameters that shift the steadystate friction curve to smaller slip velocities and to employ a different nucleation procedure.

In particular, using the different set of parameters listed in Table II, we obtain the dash-dotted orange steady-state curve in Fig. 7(a) [the solid brown line is identical to the

TABLE II. The values of the rate-and-state parameters (in MKS units), which are discussed in Fig. 7 and which gave rise to the slow rupture shown in Fig. 4.

\begin{tabular}{lcc}
\hline \hline Parameter & Value & Units \\
\hline$D$ & $5 \times 10^{-7}$ & $\mathrm{~m}$ \\
$b$ & 0.1 & $\cdots$ \\
$v_{*}$ & $10^{-8}$ & $\mathrm{~m} / \mathrm{s}$ \\
$f_{0}$ & 0.28 & $\cdots$ \\
$\phi_{*}$ & 0.05 & $\mathrm{~s}$ \\
$a$ & 0.0075 & $\cdots$ \\
\hline \hline
\end{tabular}
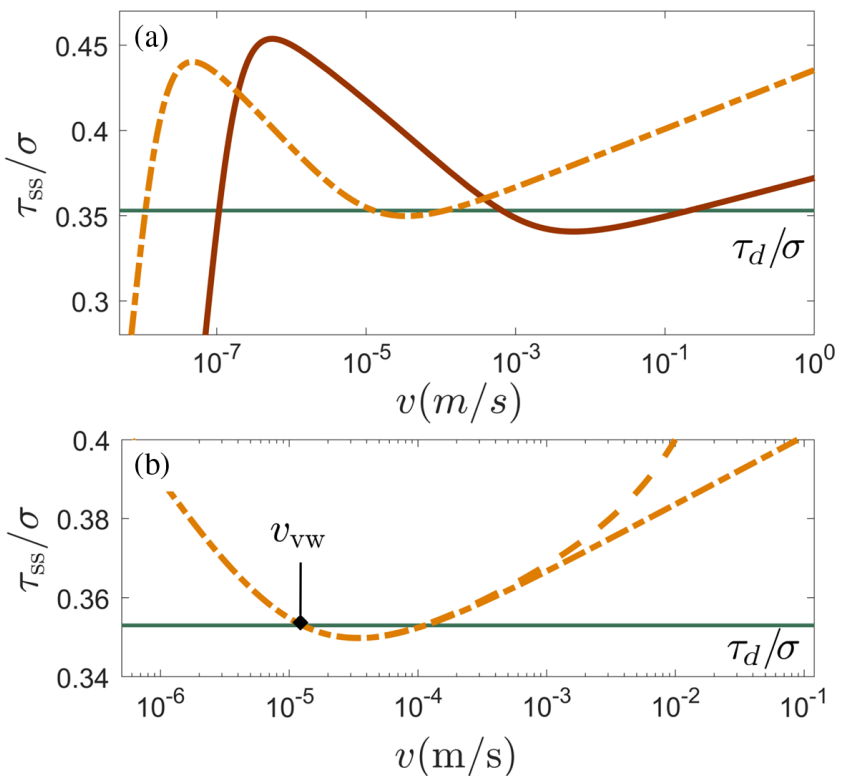

FIG. 7. (a) The normalized steady-state friction law as used in Fig. 2(a) (solid brown line) and the modified one that corresponds to the parameters listed in Table II (dash-dotted orange line). The horizontal green line represents the normalized driving stress $\tau_{d} / \sigma$. (b) An enlargement of the dash-dotted orange line in (a), where the effective steady-state friction curve (dashed orange line, obtained by adding the radiation-damping term $\left.\left(\mu / 2 c_{s}\right) v\right)$ is added. The intersection of the driving stress with the velocityweakening branch of the friction law is denoted by $v_{\mathrm{vw}}$ (black diamond). Perturbations around $v_{\mathrm{vw}}$ lead to the slow rupture shown in Fig. 4; see the text for additional details.

one shown in Fig. 2(a)]. For these parameters and for the same value of the normalized driving stress $\tau_{d} / \sigma$, the effective steady-state friction curve shown in Fig. 7(b) (dashed orange line, obtained by adding the radiationdamping term $\left.\left(\mu / 2 c_{s}\right) v\right)$ is practically indistinguishable from the steady-state friction curve in the relevant slip velocities range. In addition, the rupture is nucleated by introducing a perturbation to the internal state field $\phi$ of the form

$$
\phi(x, t=0)=\frac{D}{v_{\mathrm{vw}}}+\varepsilon \sin (k x),
$$

with $k=2 \pi / W$ and $\varepsilon=10^{-4}$, into an interface that slides homogeneously at a velocity $v_{\mathrm{vw}}$ that corresponds to a fixed point on the velocity-weakening branch [it is marked by the black diamond in Fig. 7(b)]. This nucleation procedure is different from the one used elsewhere in the paper, where Gaussian perturbations are introduced into an essentially locked-in interface, as described in detail in Ref. [80].

These modifications are sufficient to generate the slow rupture shown in Fig. 4. The physics behind the emergence of a slow rupture, which is very interesting in itself, is not thoroughly discussed here. It deserves an investigation of its own, which we hope to pursue in the future. 


\section{APPENDIX F: THE EXPERIMENTAL DATA}

The data reported in Fig. 5 are obtained from the experimental measurements [35] of ruptures propagating along a frictional interface formed by two plates of Homalite. Figure 8 of Ref. [35] reports the steady-state friction coefficient versus slip rate measured at the interface in the wake of the propagating rupture front. In terms of the notation used in this manuscript, the former is the ratio $\tau_{\text {res }} / \sigma$, while the latter corresponds to $v_{\text {res }}$. In the experiments of Ref. [35], the frictional interface is precut at an angle $\alpha$ from the principal direction of the imposed compressive stress $P$, such that

$$
\begin{gathered}
\tau_{d}=P \cos \alpha \sin \alpha, \\
\sigma=P \cos ^{2} \alpha .
\end{gathered}
$$

Rubino, Rosakis, and Lapusta [35] distinguish data measured with respectively high and low levels of accuracy. From the datasets reported in Fig. 8 of Ref. [35] and their associated boundary conditions listed in Table III, we compute the triplets $\left(\tau_{d}, \tau_{\text {res }}, v_{\text {res }}\right)$, which are then used to construct Fig. 5.

In Sec. IV, we also analyze experimental data extracted from Ref. [36] in order to test the theoretical predictions in Eqs. (6) and (7). The bulk parameters $\rho=1170 \mathrm{~kg} / \mathrm{m}^{3}$ and $c_{s}=1345 \mathrm{~m} / \mathrm{s}$, reported on in Ref. [36], are used together with $\mu=\rho c_{s}^{2}$. The stress drops $\Delta \tau$ can be read off Fig. 2(b) of Ref. [36] (all figure indices in this and the next paragraphs refer to Ref. [36]), where the shear stress distribution near the edge of both a slow (red) and a rapid (here supershear, blue) rupture is presented. For a rapid rupture, we extract from Fig. 2(b) (blue data) $\Delta \tau=1.17 \mathrm{MPa}$. The corresponding particle velocity distribution $\dot{u}_{x}(x, y=3.5 \mathrm{~mm})$, measured $3.5 \mathrm{~mm}$ above the interface, is presented in Fig. 1(d) (bottom). We use the leftmost value behind the edge, $\dot{u}_{x}(x, y=3.5 \mathrm{~mm}) \simeq 0.75 \mathrm{~m} / \mathrm{s}$, as an estimate for the tail particle velocity at the interface $\dot{u}_{x}\left(y=0^{+}\right)$, from which we estimate the residual slip velocity to be $v_{\text {res }}=$ $2 \dot{u}_{x}\left(y=0^{+}\right) \simeq 1.5 \mathrm{~m} / \mathrm{s}$. Note that the latter estimate is in very good agreement with the slip velocity reported for a rapid (supershear) rupture in Fig. 3 (blue circles). Moreover, it is also in very good agreement with the normalized real area of contact $A_{r} / A_{0} \simeq 0.6$, reported on in Fig. 1(c), which

TABLE III. Datasets from Fig. 8 of Rubino, Rosakis, and Lapusta [35], which are used in Fig. 5.

\begin{tabular}{lccc}
\hline \hline Symbols & Resolution & $P[\mathrm{MPa}]$ & $\alpha$ \\
\hline Blue dots & High & 23 & $29^{\circ}$ \\
Red dots & High & 7.4 & $29^{\circ}$ \\
Black dots & High & 12 & $24^{\circ}$ \\
Green diamonds & Low & 13.6 & $29^{\circ}$ \\
Purple diamonds & Low & 23 & $29^{\circ}$ \\
\hline \hline
\end{tabular}

according to Fig. 3(b) indeed corresponds to $v_{\text {res }}$ slightly larger than $1 \mathrm{~m} / \mathrm{s}$.

Using these estimates, we obtain

$$
\frac{2 c_{s} \Delta \tau}{\mu v_{\text {res }}}=\frac{2 \Delta \tau}{\rho c_{s} v_{\text {res }}}=\frac{2 \times 1.17 \times 10^{6}}{1170 \times 1345 \times 1.5}=0.99,
$$

in great agreement with the theoretical prediction Eq. (5) in the manuscript. Repeating this procedure for a slow rupture, we extract from Fig. 2(b) (red data) $\Delta \tau=0.242 \mathrm{MPa}$ and from Fig. 1(d) (top) $v_{\text {res }}=$ $2 \dot{u}_{x}\left(y=0^{+}\right) \simeq 2 \times 0.01=0.02 \mathrm{~m} / \mathrm{s}$. Using these estimates, we obtain

$\frac{2 c_{s} \Delta \tau}{\mu v_{\text {res }}}=\frac{2 \Delta \tau}{\rho c_{s} v_{\text {res }}}=\frac{2 \times 0.242 \times 10^{6}}{1170 \times 1345 \times 0.02} \simeq 15 \gg 1$,

in great agreement with the theoretical prediction Eq. (7).

\section{APPENDIX G: USING BENCHMARK CRACK SOLUTIONS TO ESTIMATE THE INTEGRAL CONTRIBUTION TO THE STRESS DROP}

We provide here details about the estimates of the spatiotemporal integral contribution to the stress drop presented in Sec. II B. As explained in Sec. II A, the relative magnitude of the latter, denoted by $s$, is given by $\delta v_{\text {res }} / v_{\text {res }}^{0}=\left(v_{\text {res }}-v_{\text {res }}^{0}\right) / v_{\text {res }}^{0}$. Once $\Delta \tau$ is known, $v_{\text {res }}^{0}$ is obtained through Eq. (5). Our goal is to show how $v_{\text {res }}$ can be estimated using benchmark crack solutions [78]. Consider first antiplane shear (mode-III symmetry) selfsimilar crack solutions, where the crack face displacement is given in Eq. (6.9.145) in Ref. [78] and takes the form

$u_{z}\left(x, y=0^{+}, t\right)=\frac{\tau_{d} \sqrt{\left(c_{r} t\right)^{2}-x^{2}}}{\mu \boldsymbol{E}\left(1-c_{r}^{2} / c_{s}^{2}\right)} \quad$ for $|x|<c_{r} t$,

where the notation is adapted to be consistent with the present manuscript and $\boldsymbol{E}(\cdot)$ is the complete elliptic integral of the second kind [86]. Note that the center of the crack, whose length at time $t$ is $2 c_{r} t$, is located at $x=0$.

In order to estimate $v_{\text {res }}$ based on Eq. (G1), follow these steps: (i) Replace the far-field applied shear stress $\tau_{d}$ by the stress drop $\Delta \tau$ to account for a finite frictional resistance, not included in the crack solution, (ii) take the limit $c_{r} \rightarrow c_{s}$ ( $c_{s}$ is the limiting propagation speed in mode III), because only in this limit does the frictional rupture we consider mimic self-similar propagation, (iii) focus on a region far behind the propagating tip, i.e., set $x \rightarrow 0$, which is relevant for the definition of a stress drop, (iv) take the time derivative to obtain $\dot{u}_{z}\left(x=0, y=0^{+}, t\right)$, and, finally, (v) set $v_{\text {res }}=\dot{u}_{z}\left(x=0, y=0^{+}, t\right)-\dot{u}_{z}\left(x=0, y=0^{-}, t\right)=$ $2 \dot{u}_{z}\left(x=0, y=0^{+}, t\right)$. The result reads 


$$
v_{\mathrm{res}}=\frac{2 c_{s} \Delta \tau}{\mu \boldsymbol{E}(0)} .
$$

Using Eq. (5), which implies $v_{\text {res }}^{0}=2 c_{s} \Delta \tau / \mu$, together with Eq. (G2), we obtain (as reported on in Sec. II B)

$$
\delta v_{\text {res }} / v_{\text {res }}^{0}=1 / \boldsymbol{E}(0)-1=2 / \pi-1 \approx-0.36,
$$

where $\boldsymbol{E}(0)=\pi / 2$ is used.

In order to repeat this procedure for in-plane shear (mode-II symmetry) self-similar crack solutions, we start with the crack face displacement given in Eq. (6.9.85) in Ref. [78]:

$$
u_{x}\left(x, y=0^{+}, t\right)=\frac{\tau_{d}\left(c_{s}^{2}-c_{r}^{2}\right) \sqrt{\left(c_{r} t\right)^{2}-x^{2}}}{\mu c_{d}^{2} g_{2}\left(c_{r} / c_{s}, c_{s} / c_{d}\right)}
$$

for $|x|<c_{r} t$, where the function $g_{2}(\cdot)$ is given in Eq. (6.9.87) in Ref. [78] and $c_{d}$ is the dilatational wave speed. Considering the limit $c_{r} \rightarrow c_{R}$ ( $c_{R}$ is the Rayleigh wave speed, the limiting speed for mode II) and following the procedure described above, we obtain

$$
v_{\mathrm{res}}=\frac{2 c_{R} \Delta \tau}{\mu} \frac{\left(c_{s}^{2}-c_{R}^{2}\right)}{c_{d}^{2} g_{2}\left(c_{R} / c_{s}, c_{s} / c_{d}\right)},
$$

which leads to

$$
\frac{\delta v_{\mathrm{res}}}{v_{\mathrm{res}}^{0}}=\frac{\left(c_{s}^{2}-c_{R}^{2}\right) c_{R} / c_{s}}{c_{d}^{2} g_{2}\left(c_{R} / c_{s}, c_{s} / c_{d}\right)}-1
$$

The latter is a function of $c_{s} / c_{d}$ (note that $c_{R} / c_{s}$ is also determined by $\left.c_{s} / c_{d}\right)$. Evaluating $g_{2}\left(c_{R} / c_{s}, c_{s} / c_{d}\right)$ [88] for the whole range of admissible $c_{s} / c_{d}$ values (which, in turn, depends on Poisson's ratio) and plugging in Eq. (G6) shows that $\delta v_{\text {res }} / v_{\text {res }}^{0}$ varies between -0.613 and -0.417 , as reported on in Sec. II B.

[1] I. Svetlizky, E. Bayart, and J. Fineberg, Brittle Fracture Theory Describes the Onset of Frictional Motion, Annu. Rev. Condens. Matter Phys. 10, 253 (2019).

[2] C. H. Scholz, The Mechanics of Earthquakes and Faulting (Cambridge University Press, Cambridge, England, 2002).

[3] S. Rhee, M. Jacko, and P. Tsang, The Role of Friction Film in Friction, Wear and Noise of Automotive Brakes, Wear 146, 89 (1991).

[4] S. Casado, Studying Friction while Playing the Violin: Exploring the Stickslip Phenomenon, Beilstein J. Nanotechnol. 8, 159 (2017).

[5] C. Marone, Laboratory-Derived Friction Laws and Their Application to Seismic Faulting, Annu. Rev. Earth Planet Sci. 26, 643 (1998).

[6] Y. Ben-Zion, Collective Behavior of Earthquakes and Faults: Continuum-Discrete Transitions, Progressive
Evolutionary Changes, and Different Dynamic Regimes, Rev. Geophys. 46, RG4006 (2008).

[7] M. Ohnaka, The Physics of Rock Failure and Earthquakes (Cambridge University Press, Cambridge, England, 2013).

[8] L. B. Freund, Dynamic Fracture Mechanics (Cambridge University Press, Cambridge, England, 1998).

[9] Y. Ida, Cohesive Force across the Tip of a LongitudinalShear Crack and Griffith's Specific Surface Energy, J. Geophys. Res. 77, 3796 (1972).

[10] A. C. Palmer and J. R. Rice, The Growth of Slip Surfaces in the Progressive Failure of Over-Consolidated Clay, Proc. R. Soc. A 332, 527 (1973).

[11] D. J. Andrews, Rupture Propagation with Finite Stress in Antiplane Strain, J. Geophys. Res. 81, 3575 (1976).

[12] R. Madariaga, High-Frequency Radiation from Crack (Stress Drop) Models of Earthquake Faulting, Geophys. J. Int. 51, 625 (1977).

[13] A. Cochard and J. R. Rice, Fault Rupture between Dissimilar Materials: Ill-Posedness, Regularization, and Slip-Pulse Response, J. Geophys. Res. Solid Earth 105, 25891 (2000).

[14] A. Bizzarri, M. Cocco, D. J. Andrews, and E. Boschi, Solving the Dynamic Rupture Problem with Different Numerical Approaches and Constitutive Laws, Geophys. J. Int. 144, 656 (2001).

[15] K. Uenishi and J. R. Rice, Universal Nucleation Length for Slip-Weakening Rupture Instability under Nonuniform Fault Loading, J. Geophys. Res. Solid Earth 108, 2042 (2003).

[16] J. R. Rice, C. G. Sammis, and R. Parsons, Off-Fault Secondary Failure Induced by a Dynamic Slip Pulse, Bull. Seismol. Soc. Am. 95, 109 (2005).

[17] H. S. Bhat, R. Dmowska, G. C. P. King, Y. Klinger, and J. R. Rice, Off-Fault Damage Patterns due to Supershear Ruptures with Application to the 2001 Mw 8.1 Kokoxili (Kunlun) Tibet Earthquake, J. Geophys. Res. 112, B06301 (2007).

[18] Y. Liu and N. Lapusta, Transition of Mode II Cracks from Sub-Rayleigh to Intersonic Speeds in the Presence of Favorable Heterogeneity, J. Mech. Phys. Solids 56, 25 (2008).

[19] E. M. Dunham and J. R. Rice, Earthquake Slip between Dissimilar Poroelastic Materials, J. Geophys. Res. Solid Earth 113, B09304 (2008).

[20] A. Bizzarri, How to Promote Earthquake Ruptures: Different Nucleation Strategies in a Dynamic Model with SlipWeakening Friction, Bull. Seismol. Soc. Am. 100, 923 (2010).

[21] A. Bizzarri, On the Relations between Fracture Energy and Physical Observables in Dynamic Earthquake Models, J. Geophys. Res. 115, B10307 (2010).

[22] R. C. Viesca and J. R. Rice, Nucleation of Slip-Weakening Rupture Instability in Landslides by Localized Increase of Pore Pressure, J. Geophys. Res. Solid Earth 117, 1 (2012).

[23] D. S. Kammer, V. A. Yastrebov, P. Spijker, and J.-F. Molinari, On the Propagation of Slip Fronts at Frictional Interfaces, Tribol. Lett. 48, 27 (2012).

[24] C. Liu, A. Bizzarri, and S. Das, Progression of Spontaneous In-Plane Shear Faults from Sub-Rayleigh to Compressional Wave Rupture Speeds, J. Geophys. Res. Solid Earth 119, 8331 (2014). 
[25] D. S. Kammer, M. Radiguet, J.-P. Ampuero, and J.-F. Molinari, Linear Elastic Fracture Mechanics Predicts the Propagation Distance of Frictional Slip, Tribol. Lett. 57, 23 (2015).

[26] A. Bizzarri and C. Liu, Near-Field Radiated Wave Field May Help to Understand the Style of the Supershear Transition of Dynamic Ruptures, Phys. Earth Planet. Inter. 261, 133 (2016).

[27] F. Barras, P. H. Geubelle, and J.-F. Molinari, Interplay between Process Zone and Material Heterogeneities for Dynamic Cracks, Phys. Rev. Lett. 119, 144101 (2017).

[28] R. E. Abercrombie and J. R. Rice, Can Observations of Earthquake Scaling Constrain Slip Weakening?, Geophys. J. Int. 162, 406 (2005).

[29] X. Lu, A. J. Rosakis, and N. Lapusta, Rupture Modes in Laboratory Earthquakes: Effect of Fault Prestress and Nucleation Conditions, J. Geophys. Res. Solid Earth 115, 1 (2010).

[30] X. Lu, N. Lapusta, and A. J. Rosakis, Pulse-like and Cracklike Dynamic Shear Ruptures on Frictional Interfaces: Experimental Evidence, Numerical Modeling, and Implications, Int. J. Fract. 163, 27 (2010).

[31] H. Noda, N. Lapusta, and H. Kanamori, Comparison of Average Stress Drop Measures for Ruptures with Heterogeneous Stress Change and Implications for Earthquake Physics, Geophys. J. Int. 193, 1691 (2013).

[32] I. Svetlizky and J. Fineberg, Classical Shear Cracks Drive the Onset of Dry Frictional Motion, Nature (London) 509, 205 (2014).

[33] E. Bayart, I. Svetlizky, and J. Fineberg, Fracture Mechanics Determine the Lengths of Interface Ruptures That Mediate Frictional Motion, Nat. Phys. 12, 166 (2016).

[34] I. Svetlizky, D. P. Muńoz, M. Radiguet, D. S. Kammer, J.-F. Molinari, and J. Fineberg, Properties of the Shear Stress Peak Radiated ahead of Rapidly Accelerating Rupture Fronts That Mediate Frictional Slip, Proc. Natl. Acad. Sci. U.S.A. 113, 542 (2016).

[35] V. Rubino, A. J. Rosakis, and N. Lapusta, Understanding Dynamic Friction through Spontaneously Evolving Laboratory Earthquakes, Nat. Commun. 8, 15991 (2017).

[36] I. Svetlizky, E. Bayart, G. Cohen, and J. Fineberg, Frictional Resistance within the Wake of Frictional Rupture Fronts, Phys. Rev. Lett. 118, 234301 (2017).

[37] Y. Ben-Zion and J. R. Rice, Slip Patterns and Earthquake Populations along Different Classes of Faults in Elastic Solids, J. Geophys. Res. Solid Earth 100, 12959 (1995).

[38] G. Perrin, J. R. Rice, and G. Zheng, Self-Healing Slip Pulse on a Frictional Surface, J. Mech. Phys. Solids 43, 1461 (1995).

[39] G. Zheng and J. R. Rice, Conditions under which VelocityWeakening Friction Allows a Self-Healing versus a Cracklike Mode of Rupture, Bull. Seismol. Soc. Am. 88, 1466 (1998).

[40] P. Crupi and A. Bizzarri, The Role of Radiation Damping in the Modeling of Repeated Earthquake Events, Ann. Geophys. 56, R0111 (2013).

[41] Z. Peng and J. Gomberg, An Integrated Perspective of the Continuum between Earthquakes and Slow-Slip Phenomena, Nat. Geosci. 3, 599 (2010).
[42] K. Obara and A. Kato, Connecting Slow Earthquakes to Huge Earthquakes, Science 353, 253 (2016).

[43] R. Takagi, K. Obara, and T. Maeda, Slow Slip Event within a Gap between Tremor and Locked Zones in the Nankai Subduction Zone, Geophys. Res. Lett. 43, 1066 (2016).

[44] J. Gomberg, A. Wech, K. Creager, K. Obara, and D. Agnew, Reconsidering Earthquake Scaling, Geophys. Res. Lett. 43, 6243 (2016).

[45] T. Baumberger and C. Caroli, Solid Friction from Stickslip down to Pinning and Aging, Adv. Phys. 55, 279 (2006).

[46] A. L. Ruina, Slip Instability and State Variable Friction Laws, J. Geophys. Res. 88, 10359 (1983).

[47] J. R. Rice and A. L. Ruina, Stability of Steady Frictional Slipping, J. Appl. Mech. 50, 343 (1983).

[48] C. Marone, The Effect of Loading Rate on Static Friction and the Rate of Fault Healing during the Earthquake Cycle, Nature (London) 391, 69 (1998).

[49] M. Nakatani, Conceptual and Physical Clarification of Rate and State Friction: Frictional Sliding as a Thermally Activated Rheology, J. Geophys. Res. Solid Earth 106, 13347 (2001).

[50] J. H. Dieterich, Applications of Rate- and State-Dependent Friction to Models of Fault Slip and Earthquake Occurrence, Treatise Geophys. 4, 107 (2007).

[51] K. Nagata, M. Nakatani, and S. Yoshida, A Revised Rateand State-Dependent Friction Law Obtained by Constraining Constitutive and Evolution Laws Separately with Laboratory Data, J. Geophys. Res. Solid Earth 117, B02314 (2012).

[52] P. Bhattacharya and A. M. Rubin, Frictional Response to Velocity Steps and 1-D Fault Nucleation under a State Evolution Law with Stressing-Rate Dependence, J. Geophys. Res. Solid Earth 119, 2272 (2014).

[53] J. H. Dieterich, Earthquake Nucleation on Faults with Rateand State-Dependent Strength, Tectonophysics 211, 115 (1992).

[54] M. Roy and C. Marone, Earthquake Nucleation on Model Faults with Rate- and State-Dependent Friction: Effects of Inertia, J. Geophys. Res. Solid Earth 101, 13919 (1996).

[55] Y. Ben-Zion and J. R. Rice, Dynamic Simulations of Slip on a Smooth Fault in an Elastic Solid, J. Geophys. Res. Solid Earth 102, 17771 (1997).

[56] T. Baumberger, P. Berthoud, and C. Caroli, Physical Analysis of the State- and Rate-Dependent Friction Law. II. Dynamic Friction, Phys. Rev. B 60, 3928 (1999).

[57] N. Lapusta, J. R. Rice, Y. Ben-Zion, and G. Zheng, Elastodynamic Analysis for Slow Tectonic Loading with Spontaneous Rupture Episodes on Faults with Rate- and State-Dependent Friction, J. Geophys. Res. Solid Earth 105, 23765 (2000).

[58] M. Aldam, M. Weikamp, R. Spatschek, E. A. Brener, and E. Bouchbinder, Critical Nucleation Length for Accelerating Frictional Slip, Geophys. Res. Lett. 44, 11,390 (2017).

[59] Y. Bar-Sinai, R. Spatschek, E. A. Brener, and E. Bouchbinder, On the Velocity-Strengthening Behavior of Dry Friction, J. Geophys. Res. Solid Earth 119, 1738 (2014).

[60] C. Marone, C. B. Raleigh, and C. H. Scholz, Frictional Behavior and Constitutive Modeling of Simulated Fault Gouge, J. Geophys. Res. 95, 7007 (1990). 
[61] C. H. Scholz, Earthquakes and Friction Laws, Nature (London) 391, 37 (1998).

[62] A. M. Rubin and J.-P. Ampuero, Earthquake Nucleation on (Aging) Rate and State Faults, J. Geophys. Res. Solid Earth 110, B11312 (2005).

[63] J.-P. Ampuero and A. M. Rubin, Earthquake Nucleation on Rate and State Faults-Aging and Slip Laws, J. Geophys. Res. Solid Earth 113, B01302 (2008).

[64] S. B. Nielsen, From Slow to Fast Faulting: Recent Challenges in Earthquake Fault Mechanics, Phil. Trans. R. Soc. A 375, 20160016 (2017).

[65] E. Bayart, I. Svetlizky, and J. Fineberg, Slippery but Tough: The Rapid Fracture of Lubricated Frictional Interfaces, Phys. Rev. Lett. 116, 194301 (2016).

[66] I. Svetlizky, D. S. Kammer, E. Bayart, G. Cohen, and J. Fineberg, Brittle Fracture Theory Predicts the Equation of Motion of Frictional Rupture Fronts, Phys. Rev. Lett. 118, 125501 (2017).

[67] P. Geubelle and J. R. Rice, A Spectral Method for ThreeDimensional Elastodynamic Fracture Problems, J. Mech. Phys. Solids 43, 1791 (1995).

[68] J. W. Morrissey and P. H. Geubelle, A Numerical Scheme for Mode III Dynamic Fracture Problems, Int. J. Numer. Methods Eng. 40, 1181 (1997).

[69] M. S. Breitenfeld and P. H. Geubelle, Numerical Analysis of Dynamic Debonding under 2D In-Plane and 3D Loading, Int. J. Fract. 93, 13 (1998).

[70] J. R. Rice and Y. Ben-Zion, Slip Complexity in Earthquake Fault Models, Proc. Natl. Acad. Sci. U.S.A. 93, 3811 (1996).

[71] A. Cochard and R. Madariaga, Dynamic Faulting under Rate-Dependent Friction, Pure Appl. Geophys. 142, 419 (1994).

[72] J. N. Brune, Tectonic Stress and the Spectra of Seismic Shear Waves from Earthquakes, J. Geophys. Res. 75, 4997 (1970).

[73] P. G. Okubo and J. H. Dieterich, Effects of Physical Fault Properties on Frictional Instabilities Produced on Simulated Faults, J. Geophys. Res. Solid Earth 89, 5817 (1984).

[74] J. R. Rice, Spatio-Temporal Complexity of Slip on a Fault, J. Geophys. Res. Solid Earth 98, 9885 (1993).

[75] Y. Bar Sinai, E. A. Brener, and E. Bouchbinder, Slow Rupture of Frictional Interfaces, Geophys. Res. Lett. 39, L03308 (2012).

[76] Y. Bar-Sinai, M. Aldam, R. Spatschek, E. A. Brener, and E. Bouchbinder, Spatiotemporal Dynamics of Frictional Systems: The Interplay of Interfacial Friction and Bulk Elasticity, Lubricants 7, 91 (2019)..
[77] J. Weertman, Relationship between Displacements on a Free Surface and the Stress on a Fault, Bull. Seismol. Soc. Am. 55, 945 (1965).

[78] K. B. Broberg, Cracks and Fracture (Academic, New York, 1999).

[79] H. Noda, E. M. Dunham, and J. R. Rice, Earthquake Ruptures with Thermal Weakening and the Operation of Major Faults at Low Overall Stress Levels, J. Geophys. Res. Solid Earth 114, B07302 (2009).

[80] E. A. Brener, M. Aldam, F. Barras, J.-F. Molinari, and E. Bouchbinder, Unstable Slip Pulses and Earthquake Nucleation as a Nonequilibrium First-Order Phase Transition, Phys. Rev. Lett. 121, 234302 (2018).

[81] R. P. Singh and V. Parameswaran, An Experimental Investigation of Dynamic Crack Propagation in a Brittle Material Reinforced with a Ductile Layer, Opt. Lasers Eng. 40, 289 (2003).

[82] M. Radiguet, D. S. Kammer, P. Gillet, and J.-F. Molinari, Survival of Heterogeneous Stress Distributions Created by Precursory Slip at Frictional Interfaces, Phys. Rev. Lett. 111, 164302 (2013).

[83] E. A. Brener, S. V. Malinin, and V. I. Marchenko, Fracture and Friction: Stick-Slip Motion, Eur. Phys. J. E 17, 101 (2005).

[84] Y. Bar-Sinai, R. Spatschek, E. A. Brener, and E. Bouchbinder, Instabilities at Frictional Interfaces: Creep Patches, Nucleation, and Rupture Fronts, Phys. Rev. E 88, 060403(R) (2013).

[85] W. H. Press, S. A. Teukolsky, W. T. Vetterling, and B. P. Flannery, The Art of Scientific Computing, Numerical Recipes, 3rd ed. (Cambridge University Press, Cambridge, England, 2007).

[86] Note that, according to conventional definitions of $\boldsymbol{E}(\cdot)$ (cf. Chap. 18 in Ref. [87]), one should have $\boldsymbol{E}\left(1-c_{r}^{2} / c_{s}^{2}\right)$ in Eq. (G1) [instead of $\boldsymbol{E}\left(\sqrt{1-c_{r}^{2} / c_{s}^{2}}\right)$ as appears in Eq. (6.9.87) in Ref. [78] ].

[87] G. B. Arfken, H. J. Weber, and F. E. Harris, Mathematical Methods for Physicists: A Comprehensive Guide, 7th ed. (Academic, New York, 2011).

[88] Note that $g_{2}(\cdot)$ is given in Eq. (6.9.87) in Ref. [78] in terms of $k \equiv c_{s} / c_{d}$ and $c_{r} / c_{d}$, where $c_{r} \rightarrow c_{R}$ in our case. Moreover, note that $g_{2}(\cdot)$ also contains the complete elliptic integral of the first and second kind, $\boldsymbol{K}(\cdot)$ and $\boldsymbol{E}(\cdot)$, respectively. As noted in Ref. [86], if one employs conventional definitions of the latter two (cf. Chap. 18 in Ref. [87]), then the argument of $\boldsymbol{K}(\cdot)$ and $\boldsymbol{E}(\cdot)$ in Eq. (6.9.87) in Ref. [78] should be replaced by its square. 